Supplementary Table 1. List of antibodies

\begin{tabular}{|c|c|c|c|c|}
\hline Antibody & Host & Application & $\begin{array}{l}\text { Catalog } \\
\text { number }\end{array}$ & Vendor \\
\hline anti-HSP90 & Mouse & WB & sc-13119 & Santa Cruz Biotechnology \\
\hline anti- $\alpha$-tubulin & Mouse & WB & T5168 & Sigma-Aldrich \\
\hline anti-PRMT1 & Rabbit & WB & $07-404$ & EMD Millipore \\
\hline $\begin{array}{l}\text { Total OXPHOS } \\
\text { Rodent WB }\end{array}$ & Mouse & WB & ab110413 & Abcam \\
\hline anti-AKT & Rabbit & WB & $\# 9272$ & Cell Signaling Technology \\
\hline anti-p-AKT (S473) & Rabbit & WB & \#9271 & Cell Signaling Technology \\
\hline anti-FOXO1 & Rabbit & WB & $\# 9454$ & Cell Signaling Technology \\
\hline anti-FOXO3a & Rabbit & WB & $\# 12829$ & Cell Signaling Technology \\
\hline anti-PRMT6 & Rabbit & WB & $\# 14641$ & Cell Signaling Technology \\
\hline anti-LC3A/B & Rabbit & WB & \#4108 & Cell Signaling Technology \\
\hline $\begin{array}{l}\text { Autophagy } \\
\text { sampler kit }\end{array}$ & Rabbit & WB & $\# 4445$ & Cell Signaling Technology \\
\hline anti-AMPK $\alpha$ & Rabbit & WB & $\# 2532$ & Cell Signaling Technology \\
\hline $\begin{array}{l}\text { anti-p-AMPK } \alpha \\
\text { (T172) }\end{array}$ & Rabbit & WB & $\# 2535$ & Cell Signaling Technology \\
\hline anti-HSL & Rabbit & WB & \#4107 & Cell Signaling Technology \\
\hline anti-p-HSL (S660) & Rabbit & WB & $\# 4126$ & Cell Signaling Technology \\
\hline anti-UCP1 & Rabbit & WB & ab10983 & Abcam \\
\hline $\begin{array}{l}\text { ANTI-FLAG® } \\
\text { M2-Peroxidase } \\
\text { (HRP) antibody }\end{array}$ & Mouse & WB & A8592 & Sigma-Aldrich \\
\hline $\begin{array}{l}\text { PE anti-mouse } \\
\text { CD140a Antibody }\end{array}$ & Rat & Cell isolation & 135905 & BioLegend \\
\hline $\begin{array}{l}\text { Anti-PE } \\
\text { MicroBeads }\end{array}$ & Mouse & Cell isolation & $130-048-801$ & Miltenyi Biotec \\
\hline $\begin{array}{l}\text { anti-Pyruvate } \\
\text { Dehydrogenase }\end{array}$ & Mouse & IF & ab110333 & Abcam \\
\hline anti-F4/80 & Rat & IHC & ab6640 & Abcam \\
\hline
\end{tabular}




\begin{tabular}{|l|l|l|l|l|}
\hline anti-LC3B & Mouse & IF & $\# 83506$ & Cell Signaling Technology \\
\hline anti-Perilipin-1 & Rabbit & IF & $\# 9349$ & Cell Signaling Technology \\
\hline $\begin{array}{l}\text { Alexa Fluor® 568 } \\
\text { Goat Anti-Rabbit } \\
\text { (IgG) secondary } \\
\text { antibody }\end{array}$ & Goat & IF & A11011 & Invitrogen \\
\hline $\begin{array}{l}\text { Alexa Fluor } \\
\text { Goat Anti-Mouse } \\
\text { (IgG) secondary } \\
\text { antibody }\end{array}$ & Goat & IF & A11001 & Invitrogen \\
\hline anti-rat IgG-HRP & Goat & IF & sc-2006 & Santa Cruz Biotechnology \\
\hline Anti-FOXO3a & Rabbit & ChIP & $\# 2497$ & Cell Signaling Technology \\
\hline
\end{tabular}




\section{Supplementary Fig. Legends.}

\section{Supplementary Fig. 1. Adipocyte-specific depletion of Prmt1 eliminates expression of}

Prmt1 in mature adipocytes.

A. Prmt 1 mRNA levels in mature adipocytes (M.A) and stromal vascular fraction (SVF) from 2-month-old C57BL/6 mice with $\mathrm{NCD}$ ( $\mathrm{n}=4$ and $\mathrm{n}=5$, respectively). Relative mRNA expression was normalized to L32 expression. B. Western blot analysis showing PRMT1 protein levels from various adipose tissues as well as the liver and the skeletal muscle from Prmt1 f/f and FKO mice (n=3/group). C. Western blot analysis showing PRMT1 protein levels in mature adipocytes from eWAT and iWAT. Representative blot from three independent experiments was shown. Data in A represent mean \pm SEM. ***; $\mathrm{P}<0.001$.

\section{Supplementary Fig. 2. Depletion of Prmt1 does not affect glucose and lipid metabolism} under normal chow diet conditions.

A-C. Body weight (A), organ weight (as a percentage of body weight) (B), and fat and lean mass (C) in 4- month-old Prmtl f/f and FKO mice fed normal chow diet (NCD) were shown (n=6-8/group). D-F. Ad libitum fed (D), $6 \mathrm{hr}$ fasted (E), and overnight fasted (O/N, $16 \mathrm{hr})(\mathbf{F})$ blood glucose levels from 4-month-old Prmtl f/f and FKO mice under NCD (n=6-8/group). $\mathbf{G}$ and $\mathbf{H}$. Plasma non-esterified fatty acids levels $(\mathbf{G})$ and plasma triglyceride levels $(\mathbf{H})$ in 6 hr-fasted 4-month-old Prmt 1 f/f and FKO mice under NCD (n=5-6/group). I. Insulin tolerance test (ITT) of 4-month-old Prmtl f/f and FKO mice under NCD. ITT was conducted after the intraperitoneal injection of insulin $(0.5 \mathrm{units} / \mathrm{kg})$ on $6 \mathrm{hr}$-fasted mice ( $\mathrm{n}=6-8 /$ group). Data in A-I represent mean \pm SEM. *; P < 0.05, **; P < 0.01 .

\section{Supplementary Fig. 3. Adipocyte specific Prmt1 KO mice shows reduced eWAT.}

A-G. Representative images showing eWAT (A), iWAT (B), rpWAT (C), pWAT (D), BAT (E), Skeletal muscle (F), and Liver $(\mathbf{G})$ from Prmt1 f/f and FKO mice fed HFD for 16 weeks. (H) Body weight, fat, and lean mass of Prmtl f/f and FKO mice fed HFD for 10 weeks $(\mathrm{n}=8$ 13/group). Data in $\mathbf{H}$ represent mean \pm SEM.

\section{Supplementary Fig. 4. Adipocyte-specific Prmt1 KO mice did not greatly affect adipocyte size in iWAT.}

A. Representative images of hematoxylin and eosin-stained sections from iWAT depots of Prmt $1 \mathrm{f} / \mathrm{f}$ and FKO mice with 12 weeks of high-fat feeding. Scale bar $=250 \mu \mathrm{m}$. B. Quantification of number and size of adipocytes from iWAT of Prmtl f/f and FKO mice with 12 weeks of high-fat feeding. Data in B represent mean \pm SEM.

\section{Supplementary Fig. 5. Prmt1 deficiency does not greatly affect lipolysis.}

A. Relative mRNA levels of genes encoding lipases (ATGL, HSL) in eWAT (left) and iWAT (right) from Prmt1 f/f and FKO mice fed HFD for 12 weeks (n=3-6/group). B. Protein levels of HSL and p-HSL in eWAT (left) and iWAT (right) from Prmt $1 \mathrm{f} / \mathrm{f}$ and FKO mice fed HFD for 12 weeks ( $\mathrm{n}=6 /$ group). C. Released glycerol levels from explants of eWAT (left) and iWAT (right) of Prmt $1 \mathrm{f} / \mathrm{f}$ and FKO mice fed HFD for 20 weeks (n=4/group). Lipolysis was stimulated with $10 \mu \mathrm{M}$ forskolin (Fsk) for $2 \mathrm{hr}$. Glycerol levels were normalized to tissue weight. Data in $\mathbf{A}$ and $\mathbf{C}$ represent mean \pm SEM. *; $\mathrm{P}<0.05$, n.s.: not significant. 
Supplementary Fig. 6. Depletion of Prmt1 enhanced expression of AMPK

A. Relative mRNA levels of Prkaal and Prkaa2 in 3T3-L1 adipocytes expressing either the control virus (Ad-US) or shRNA against Prmt1 (Ad-Prmt1i). (n=3/group). mRNA levels were normalized by $L 32$ expression. B. PRMT1, PRMT6, FOXO3, AMPK and p-AMPK levels in 3T3-L1 adipocytes expressing either the control virus (Ad-US) or shRNA against Prmt1 (Ad-Prmt1i). Representative data were shown. C. Relative mRNA levels of Prmt isoforms in mature adipocytes of eWAT from Prmtl f/f and FKO mice fed HFD for 10 weeks (n=3-4/group). D. Protein levels of PRMT6 and FOXOs in eWAT from Prmtl f/f and FKO mice fed HFD for 16 weeks (n=4-5/group). E. Relative mRNA levels of Prmt1, Prmt6, Foxol, and Foxo3 in 3T3-L1 adipocytes expressing either the control virus (Ad-US) or shRNA against Prmt1 (Ad-Prmtli). (n=3/group). mRNA levels were normalized by L32 expression. F. p-AMPK $\alpha$ and AMPK $\alpha$ levels in 3T3-L1 adipocytes with ectopic expression of PRMT6 or FOXO3. Data in A, C, and $\mathbf{E}$ represent mean \pm SEM. **; $\mathrm{P}<0.01$, ***; $\mathrm{P}<$ 0.001 .

\section{Supplementary Fig. 7. Depletion of Prmt1 promotes an increased expression of components in the autophagy.}

A. Relative mRNA levels of autophagy markers in mature adipocytes of eWAT from Prmt 1 $\mathrm{f} / \mathrm{f}$ and FKO mice fed HFD for 10 weeks ( $\mathrm{n}=3$-4/group). mRNA levels were normalized by L32. B. Quantitation of proteins involved in the autophagy shown in Fig. 4B. Data in A and $\mathbf{B}$ represent mean \pm SEM. *; $\mathrm{P}<0.05, * * ; \mathrm{P}<0.01$, ***; $\mathrm{P}<0.001$.

Supplementary Fig. 8. Depletion of Prmt1 leads to the enhanced autophagic flux.

A. Quantitation of LC3-II/LC3-I ratio for Fig. 4C (top) and 4D (bottom). B. Relative LC3-II levels with injection of lysosomal protease inhibitor leupeptin in Liver (top) or BAT (bottom) of Prmt1 f/f mice and FKO mice. C. Co-localization of LC3B and PLIN1 in iWAT of Prmt1 $\mathrm{f} / \mathrm{f}$ mice and FKO mice. Scale bar $=20 \mu \mathrm{m}$. D. Quantitation of fluorescent intensity to measure co-localization of LC3B and PLIN1 from eWAT (Fig. 4E) and iWAT (Supplementary Fig. 8C). Data in A and D represent mean \pm SEM. *; P < 0.05, ***; P < 0.001 .

\section{Supplementary Fig. 9. Prmt1 deficiency promotes increased mitochondrial function in adipocytes.}

A. Quantitation of the intensity of mitotracker staining in eWAT (Fig. 5B). B. Measurement of relative mitochondrial DNA (mtDNA) in eWAT (top) and iWAT (bottom) from Prmt1 f/f and FKO mice fed HFD for 16 weeks ( $\mathrm{n}=4$-5/group). Relative mtDNA was normalized to nuclear DNA (nDNA, H19). C. Relative mRNA levels of mitochondrial markers in eWAT (left) and iWAT (right) from Prmtl f/f and FKO mice after 12 weeks of high-fat feeding ( $\mathrm{n}=3$-6/group). mRNA levels were normalized by $L 32$ expression. Data in A-C represent mean \pm SEM. *; P < 0.05, **; P < 0.01, ***; P < 0.001.

Supplementary Fig. 10. Prmt1 deficiency promotes increased mitochondrial function in WAT but not in BAT.

A-D. OxPhos protein levels in rpWAT (A), pWAT (B), iWAT (C) or BAT (D) from Prmt1 $\mathrm{f} / \mathrm{f}$ and FKO mice after 12 weeks of high-fat feeding ( $\mathrm{n}=5-7 /$ group). 
Supplementary Fig. 11. Prmt1 deficiency promotes increased lipid catabolism via an AMPK pathway.

A. PRMT1, AMPKa, p-AMPK, LC3, and OxPhos protein levels in differentiated 3T3-L1 cells. Cells were transfected with control siRNA, Prmt1 siRNA, Prkaa, or Prmt1 siRNA + Prkaa siRNA before the initiation of differentiation. Representative data from three independent experiments were shown. B. PRMT1, AMPK $\alpha$, p-AMPK, LC3, and OxPhos protein levels in differentiated primary adipocytes (derived from the AP cells of eWAT) from Prmtl f/f mice. Cells were transfected with control siRNA, Prmt1 siRNA, Prkaa, or Prmt1 siRNA + Prkaa siRNA before the initiation of differentiation. Representative data from three independent experiments were shown.

\section{Supplementary Fig. 12. Prmt1 deficiency does not affect the intrinsic lipid catabolism in BAT.}

A. Western blot analysis showing UCP1 protein levels in BAT (top) or iWAT (bottom) from Prmt1 f/f and FKO mice after 12 weeks of high-fat feeding (n=3-6/group). B. Representative images of hematoxylin and eosin-stained sections from BAT depots of Prmt1 f/f and FKO mice with 12 weeks of HFD feeding under the room temperature (RT) or cold stress. Scale bar $=250 \mu \mathrm{m}$.

Supplementary Fig. 13. Prmt1 deficiency promotes an increased energy expenditure. A-D. Indirect calorimetric analysis using Prmtl f/f and FKO mice with 11 weeks of HFD feeding. Body weight (A), Regression-adjusted energy expenditure for body weight (B), Regression-adjusted energy expenditure for food intake $(\mathbf{C})$, and food intake $(\mathbf{D})$ were shown ( $\mathrm{n}=6$ /group). Data in A-D represent mean \pm SEM. **; $\mathrm{P}<0.01, * * * ; \mathrm{P}<0.001$.

\section{Supplementary Fig. 14. Adipocyte-specific Prmt1 KO mice promotes adipose tissue inflammation.}

A. Representative images of F4/80 immunohistochemistry of eWAT depots (top) or iWAT depots (bottom) from Prmt1 f/f and FKO mice with 12 weeks of high-fat feeding. Scale bar $=$ $100 \mu \mathrm{m}$. B. Relative mRNA levels of classic macrophage (M1) and alternative macrophage (M2) markers of eWAT from Prmt1 f/f and FKO mice fed HFD for 12 weeks (n=4-6/group). mRNA levels were normalized to $L 32$ expression. C. Plasma IL-6 levels and plasma TNF $\alpha$ levels from Prmt1 f/f and FKO mice with 14 or 12 weeks of high-fat feeding (n=4-6/group). Data in $\mathbf{B}$ and $\mathbf{C}$ represent mean \pm SEM. *; $\mathbf{P}<0.05$.

\section{Supplementary Fig. 15. Adipocyte-specific Prmt1 deficiency alters glucose and lipid metabolism.}

A. Ad libitum fed blood glucose levels from Prmt1 f/f and FKO mice fed HFD for 9-10 weeks ( $\mathrm{n}=11-12 /$ group). B. Plasma insulin levels in $6 \mathrm{hr}$-fasted of Prmt1 f/f and FKO mice fed HFD for 14 weeks (n=7-8/group). C. Plasma non-esterified fatty acids levels in $6 \mathrm{hr}$ fasted Prmtl f/f and FKO mice fed HFD for 12 weeks (n-5-6/groups). D. Relative mRNA levels of genes involved in lipid metabolism in the liver from Prmtl f/f and FKO mice fed HFD for 12 weeks (n=5-6/group). Relative mRNA expression was normalized to $L 32$ expression. E. Representative western blot analysis for AKT and p-AKT levels in BAT (left), iWAT (middle) and skeletal muscle (right) from Prmt1 f/f and FKO mice fed HFD for 10 weeks. Levels of p-AKT (serine 473), total AKT, PRMT1, and loading control (HSP90) in the absence or in the presence of insulin injection were shown. All tissues were collected 
from mice post PBS or insulin (0.2 units/mouse) injection. F. Quantitation of p-AKT over AKT in BAT (left), iWAT (middle) and skeletal muscle (right) from Prmt1 f/f and FKO mice fed HFD for 10 weeks (n=3/group). Data in A-D and $\mathbf{F}$ represent mean \pm SEM. *; $\mathrm{P}<0.05$, $* * ; \mathrm{P}<0.01$.

\section{Supplementary Fig. 16. Proposed model for PRMT-mediated regulation of lipid homeostasis.}

In wild-type mice (left), obesity induces expression of PRMT1, inhibiting excessive lipid catabolism in WAT to prevent unnecessary ectopic accumulation of lipid in the peripheral tissues. In the absence of adipose PRMT1 (right), lipid catabolism in WAT is enhanced via the activation of AMPK and subsequent induction of lipophagy as well as mitochondrial catabolism, leading to the redistribution of lipid in the peripheral tissues and the resultant dysregulation of glucose and lipid homeostasis in the diet-induced obesity setting. 


\section{Supplementary Fig. 1}

A

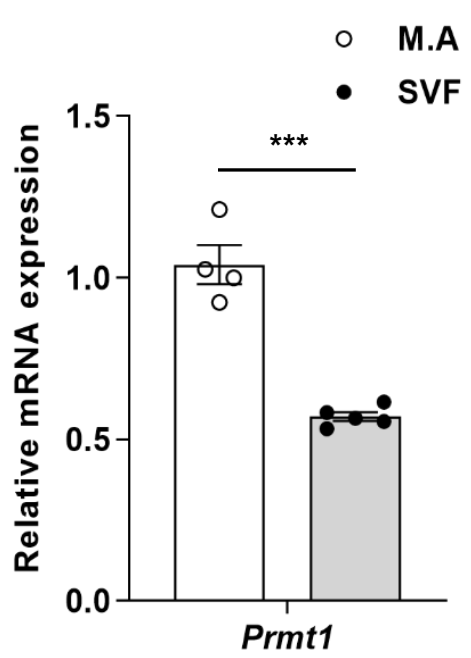

C

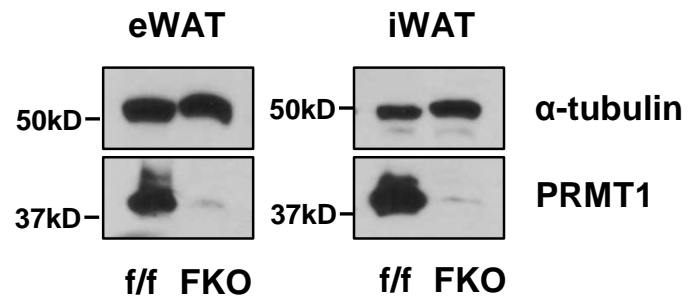

B
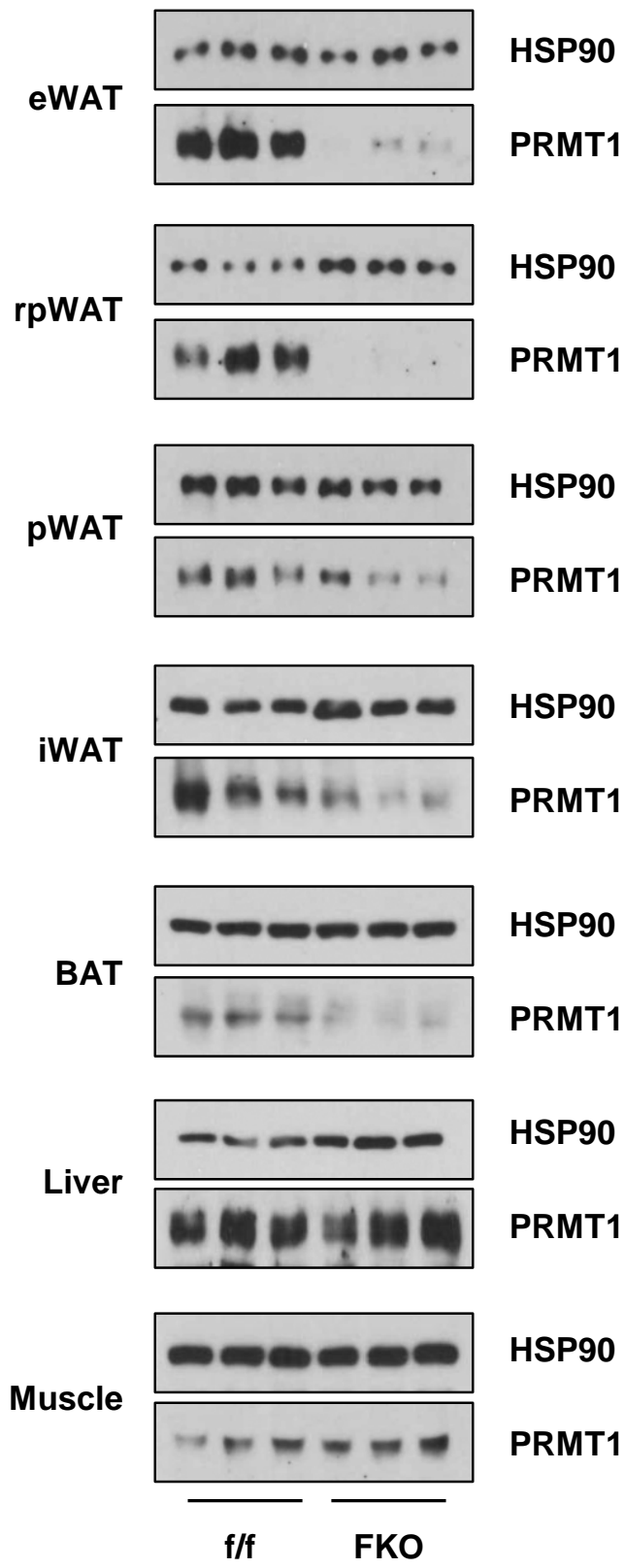
A

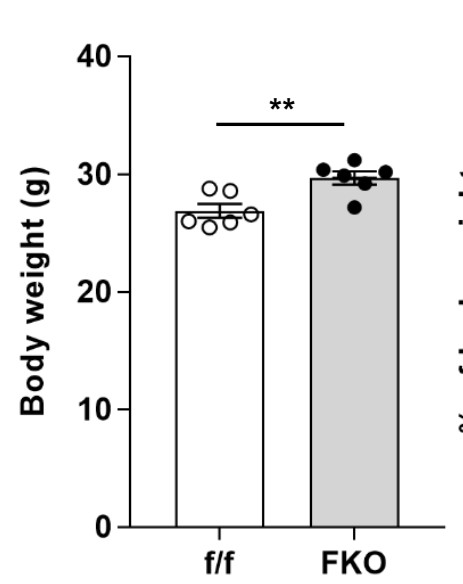

D

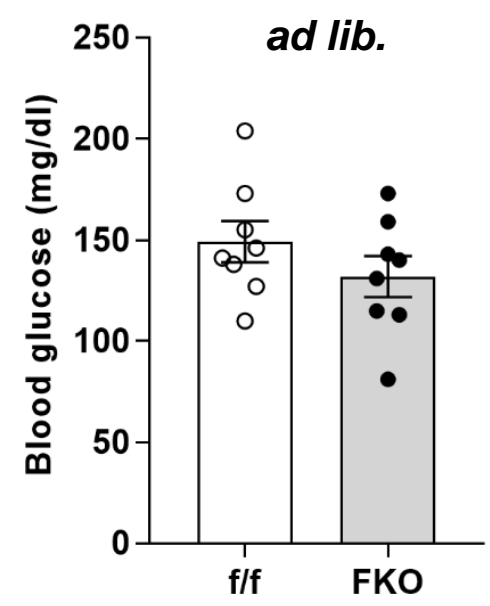

B

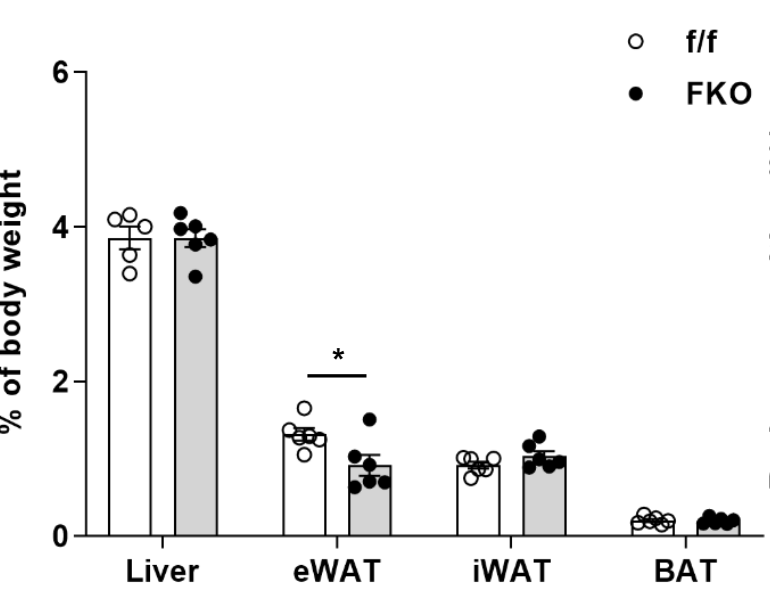

E

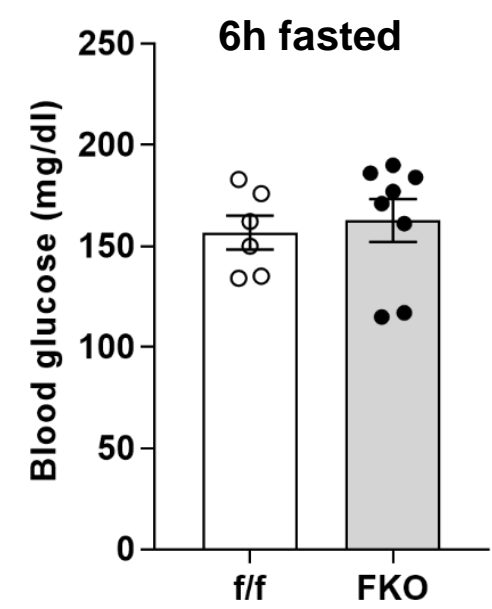

C

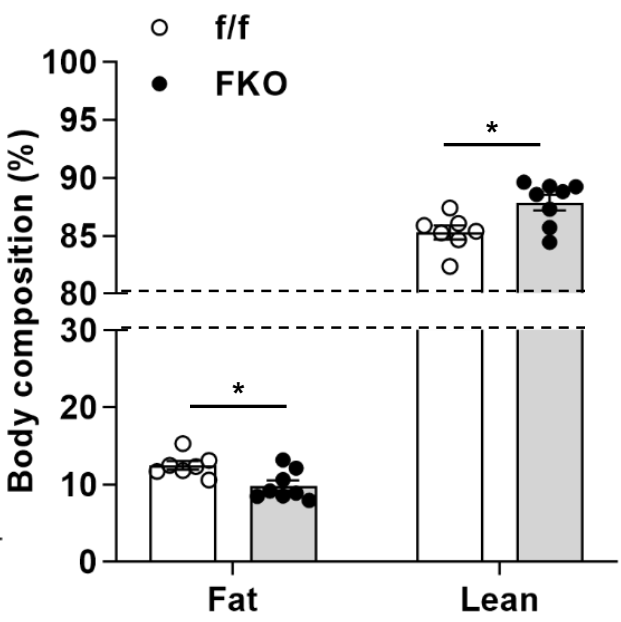

F

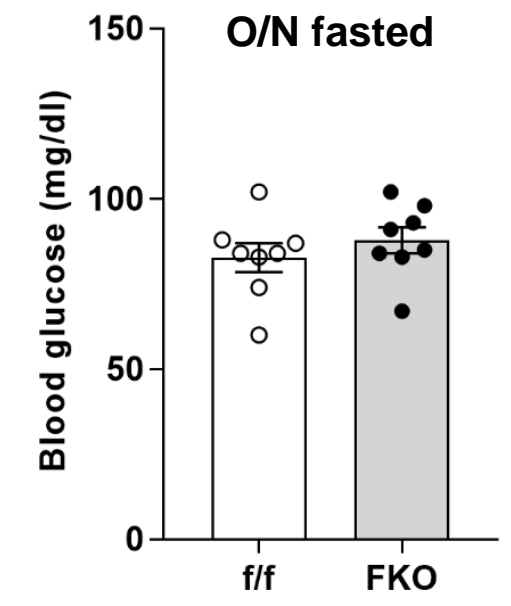

G

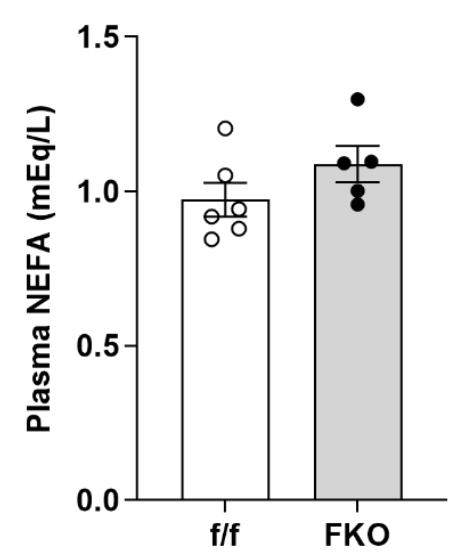

H

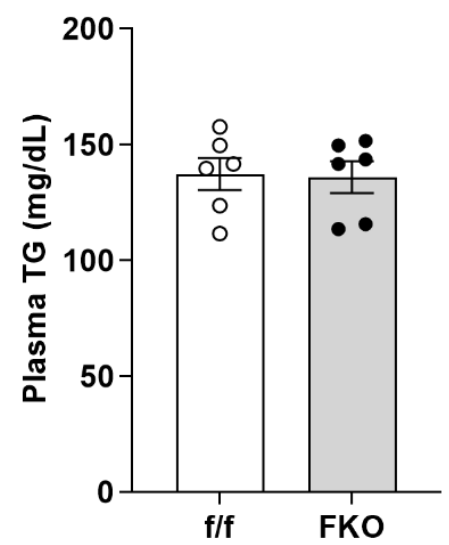

I

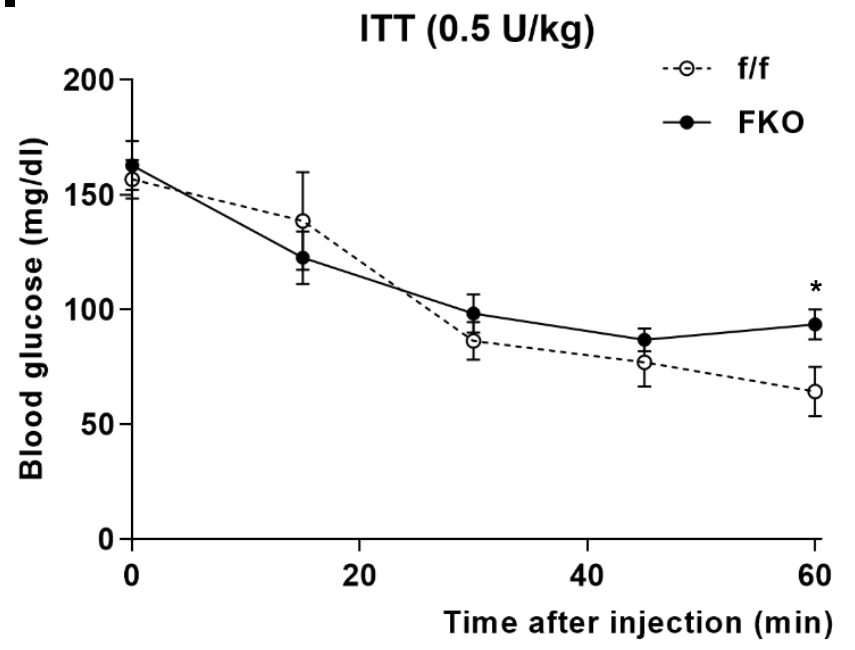




\section{Supplementary Fig. 3}

A eWAT

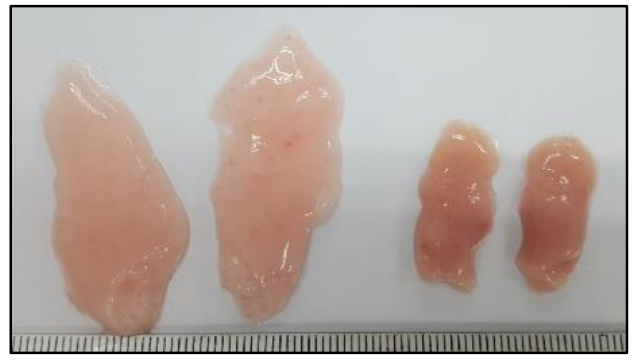

$\mathbf{f} / \mathbf{f}$

C rpWAT

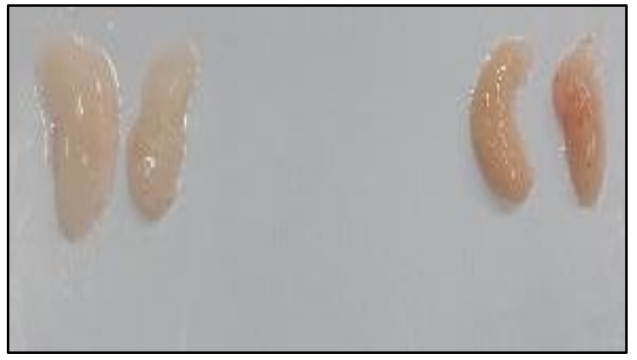

E

$\mathbf{f} / \mathbf{f}$

BAT

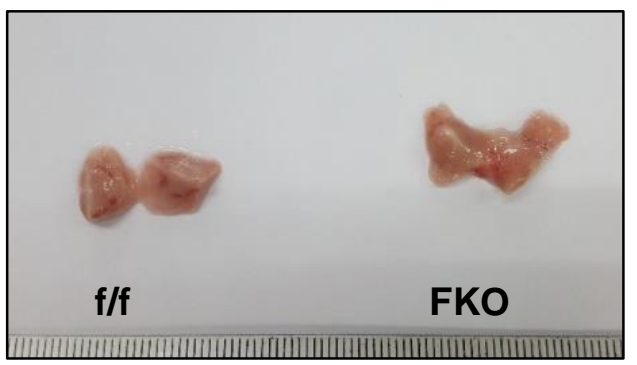

G
Liver
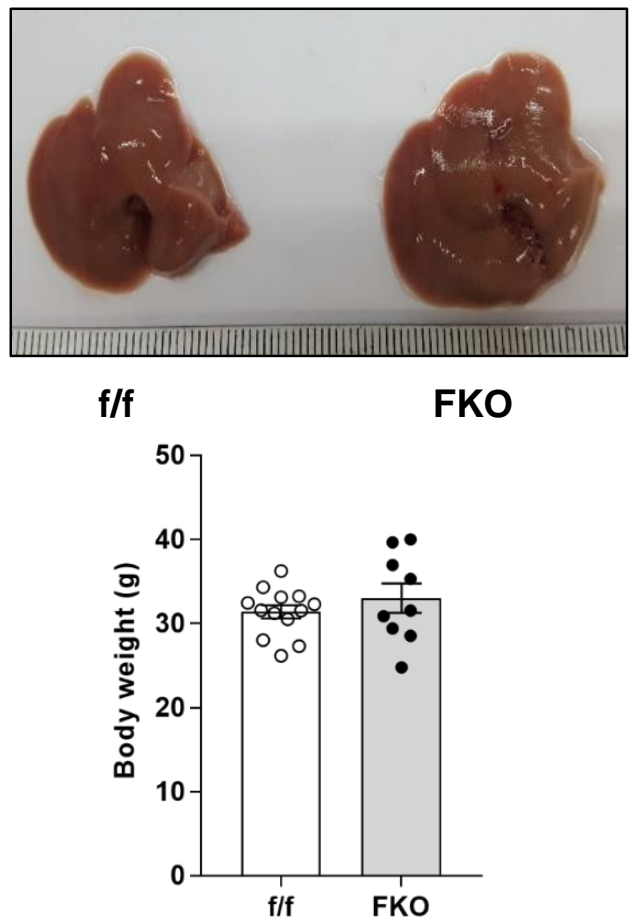

B IWAT

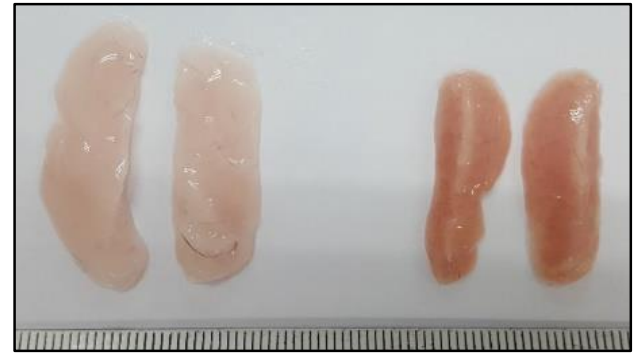

$\mathbf{f} / \mathbf{f}$

FKO

D pWAT

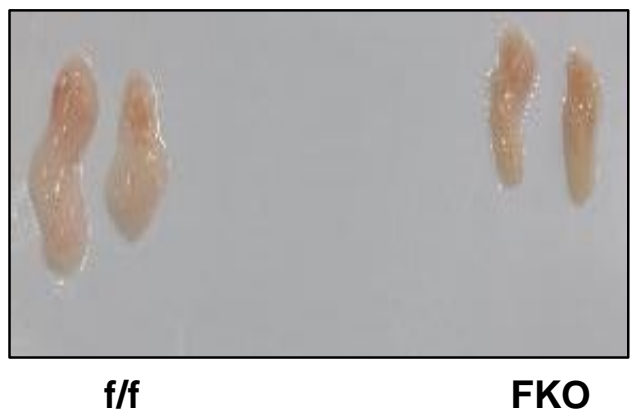

F

Muscle

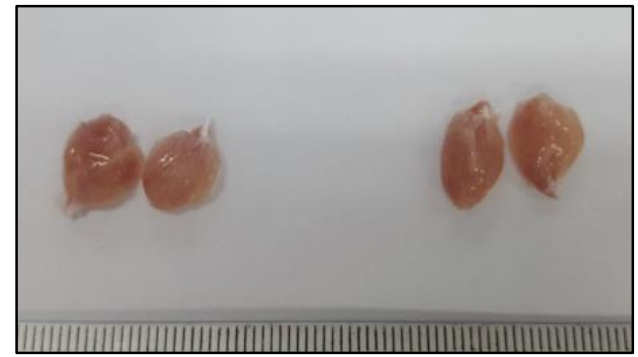

f/f

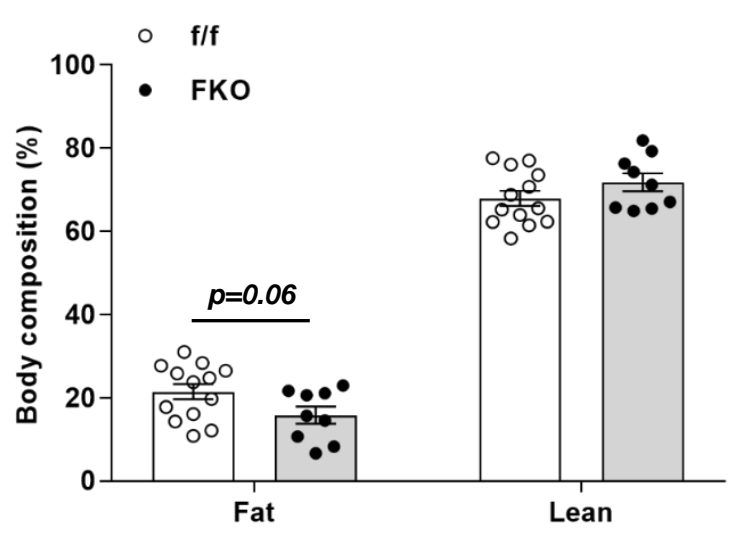




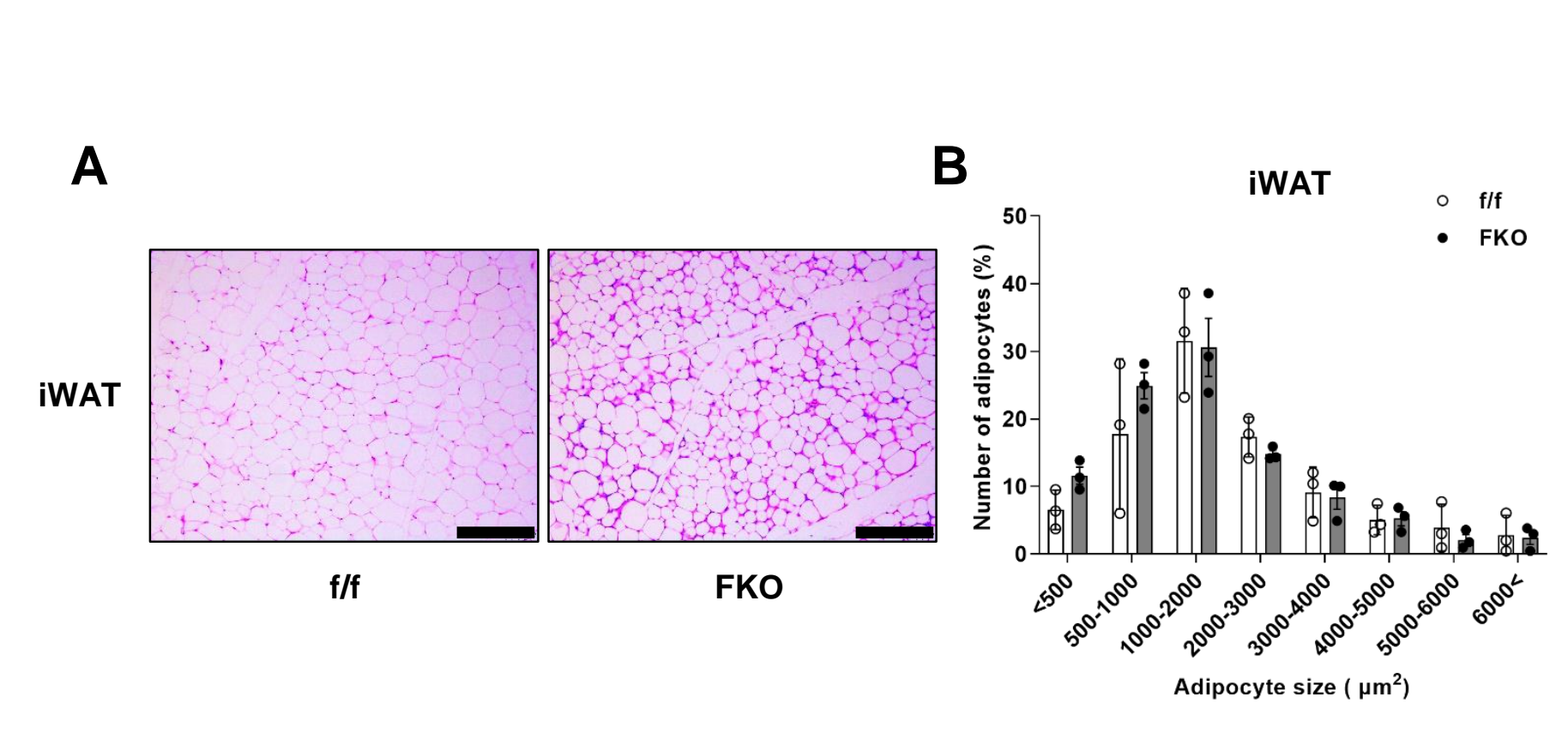

\section{Supplementary Fig. 4}
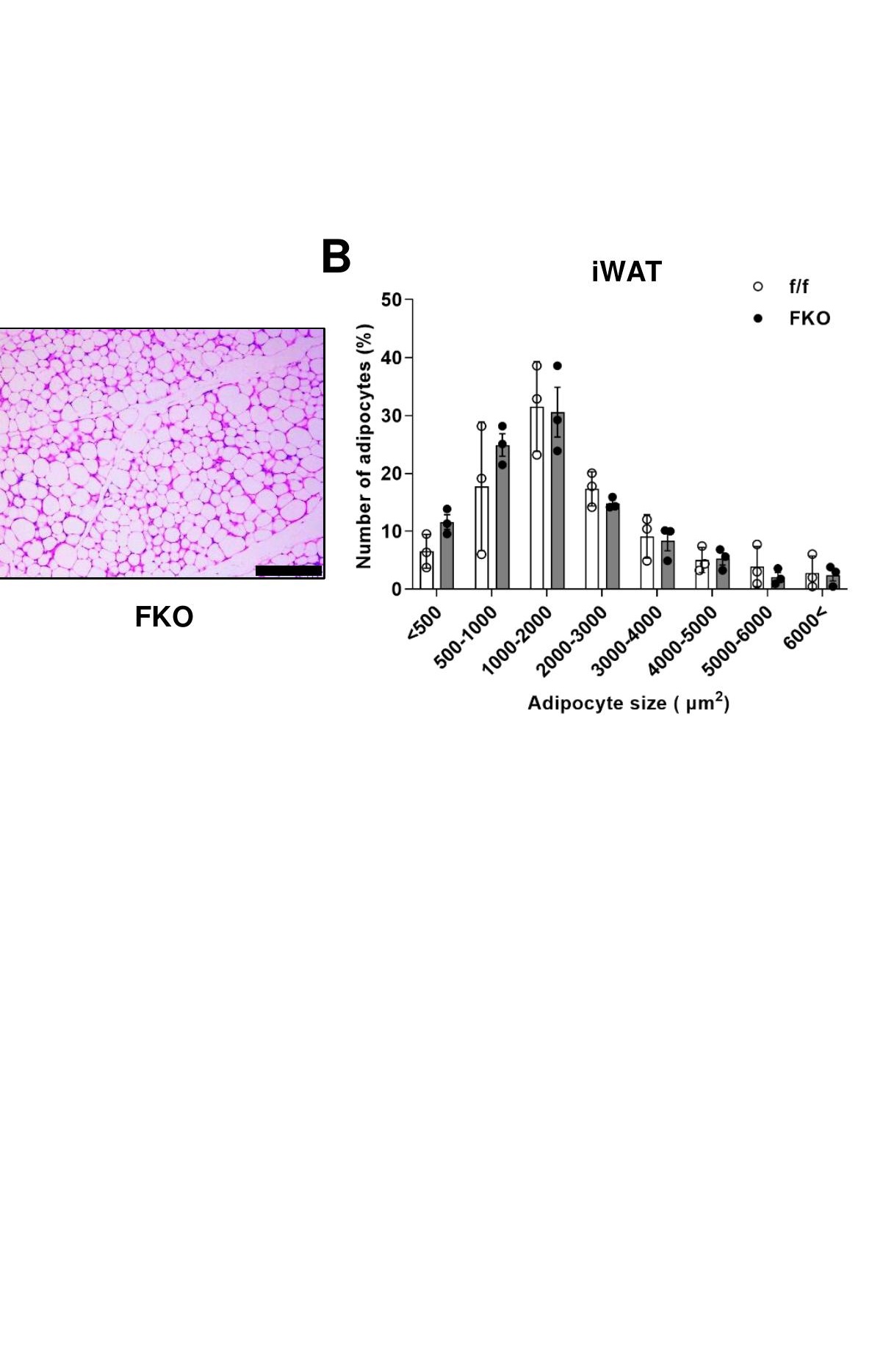


\section{Supplementary Fig. 5}

A
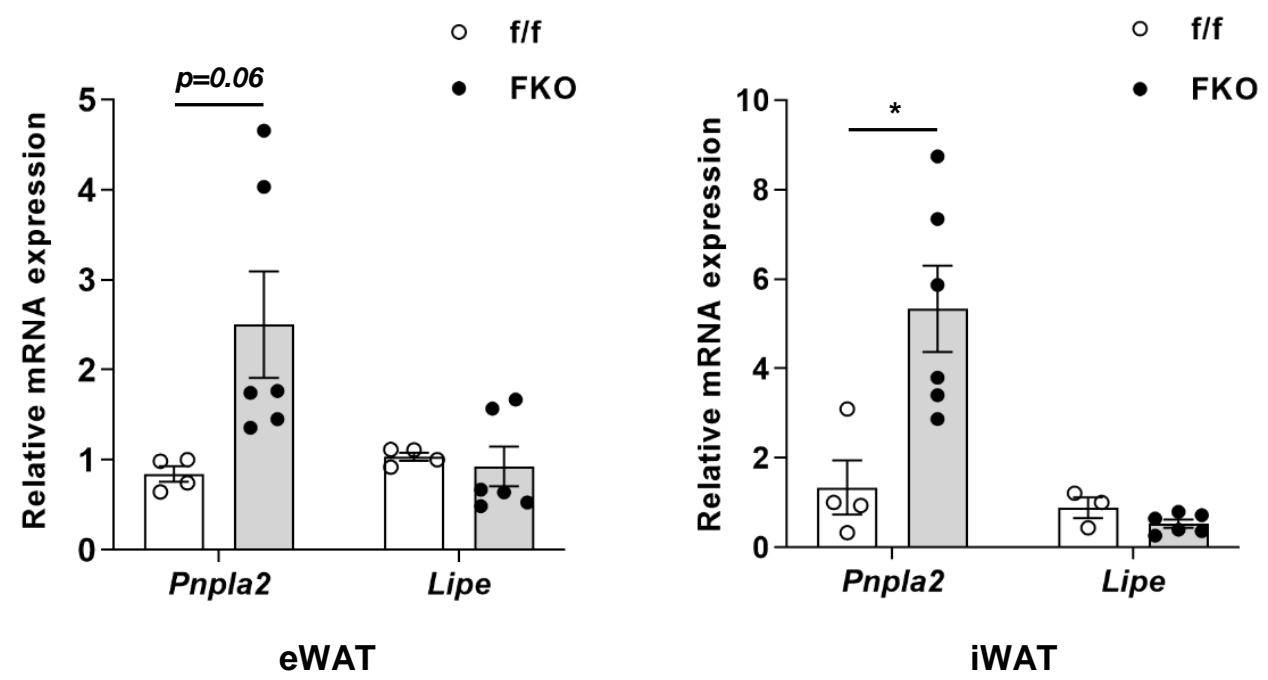

B
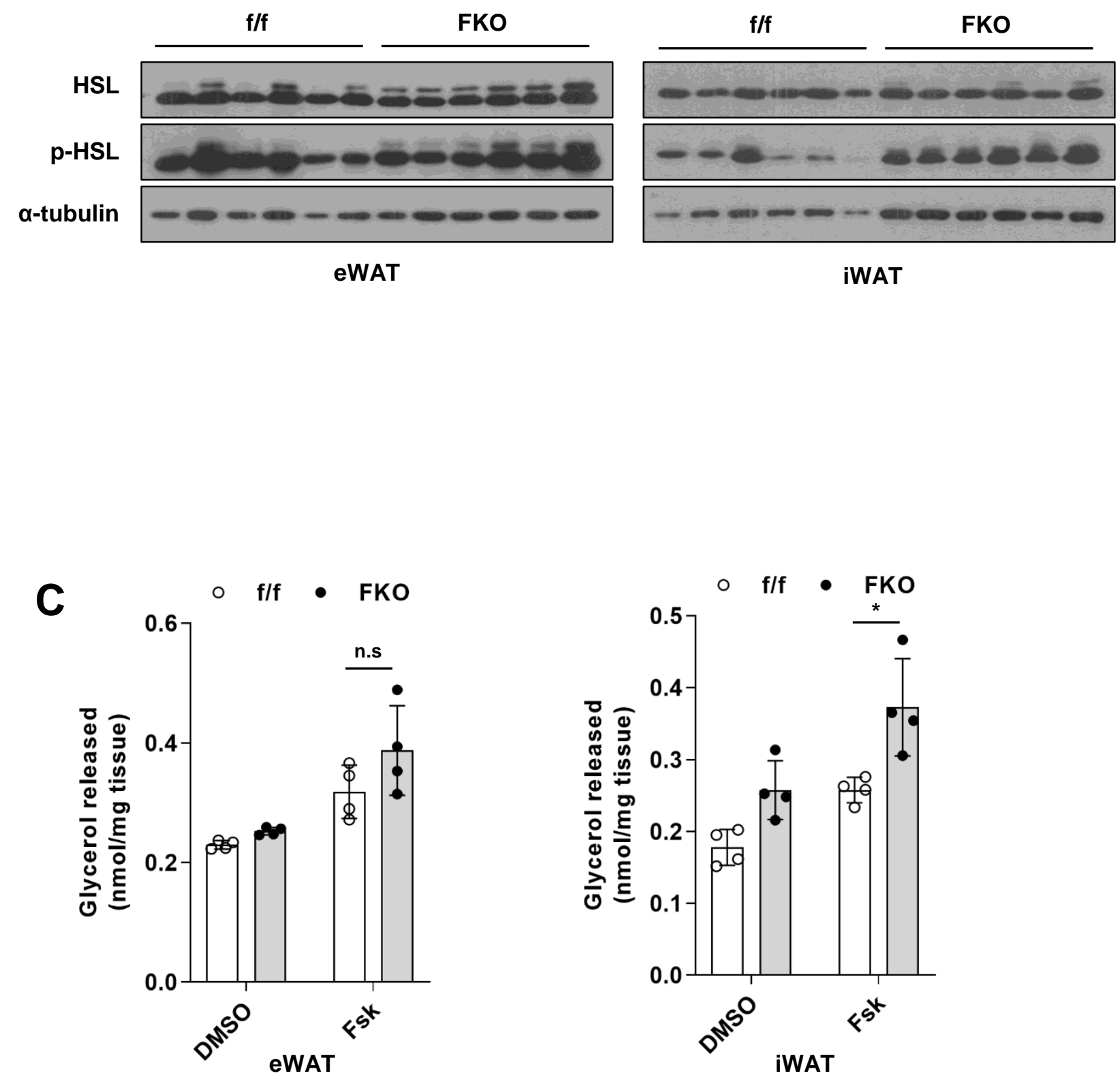


\section{Supplementary Fig. 6}

A

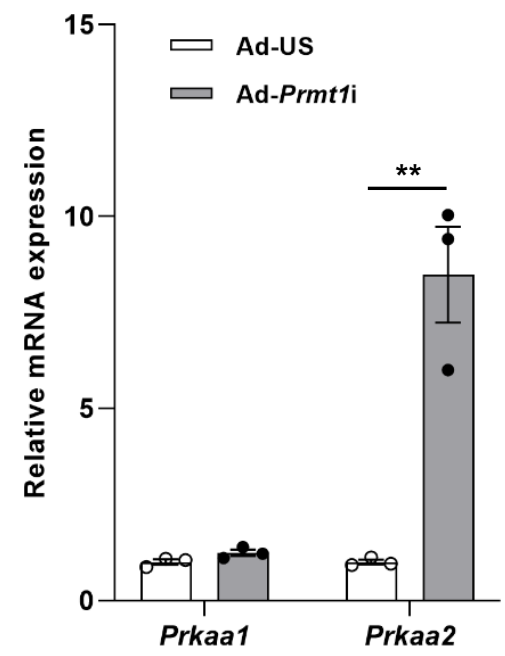

C

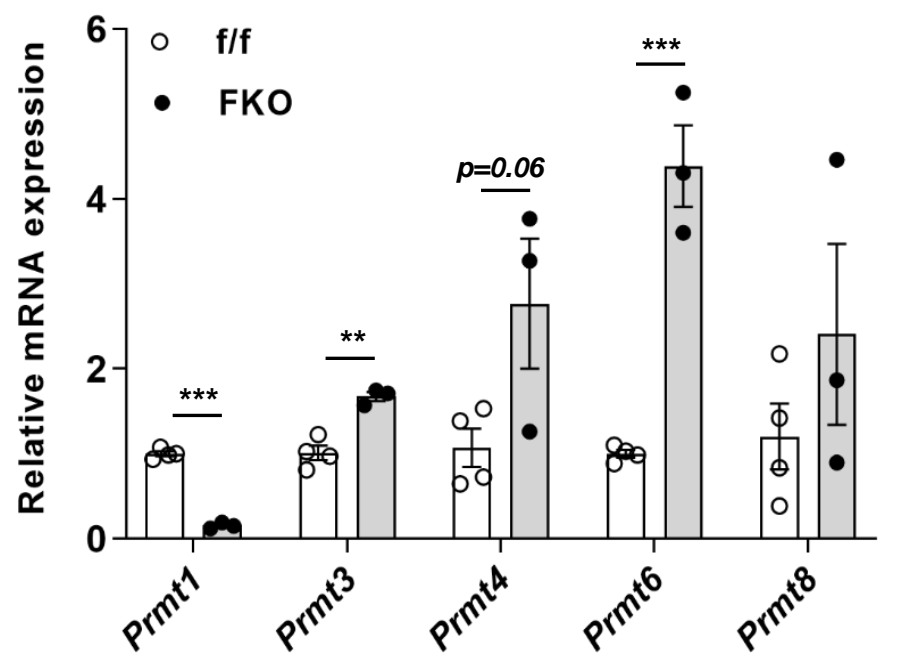

E

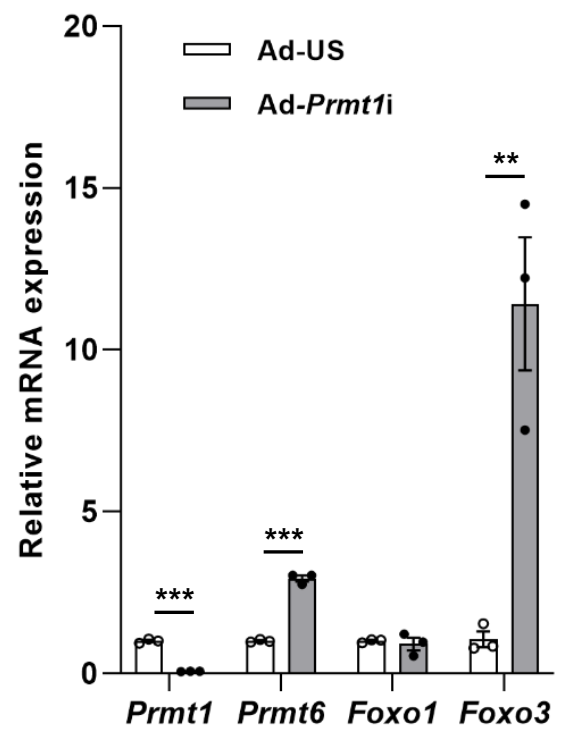

B

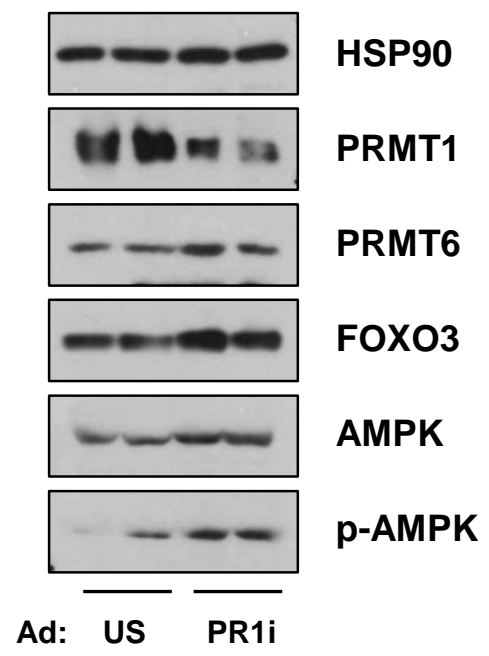

D

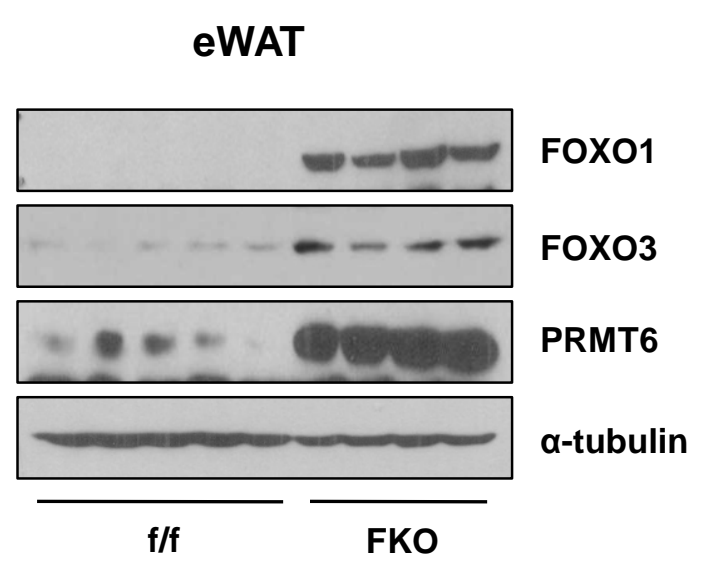

F

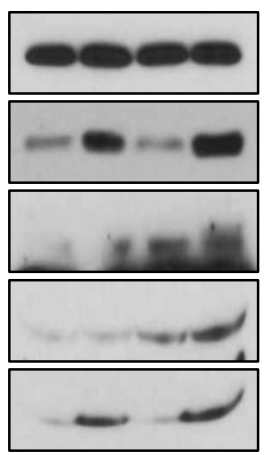

HSP90

FOXO3

Flag (PRMT6)

AMPK

p-AMPK

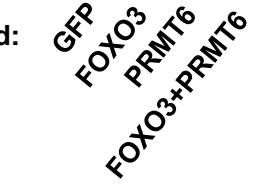


A

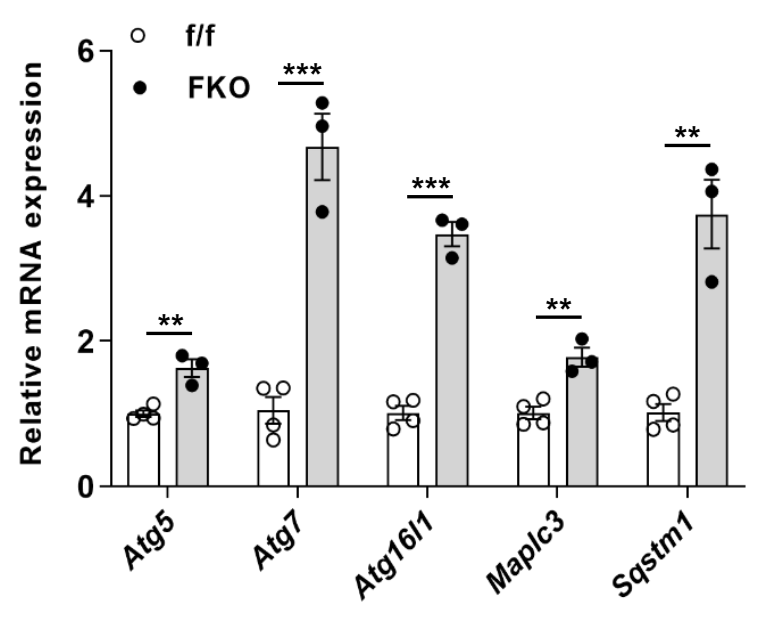

B
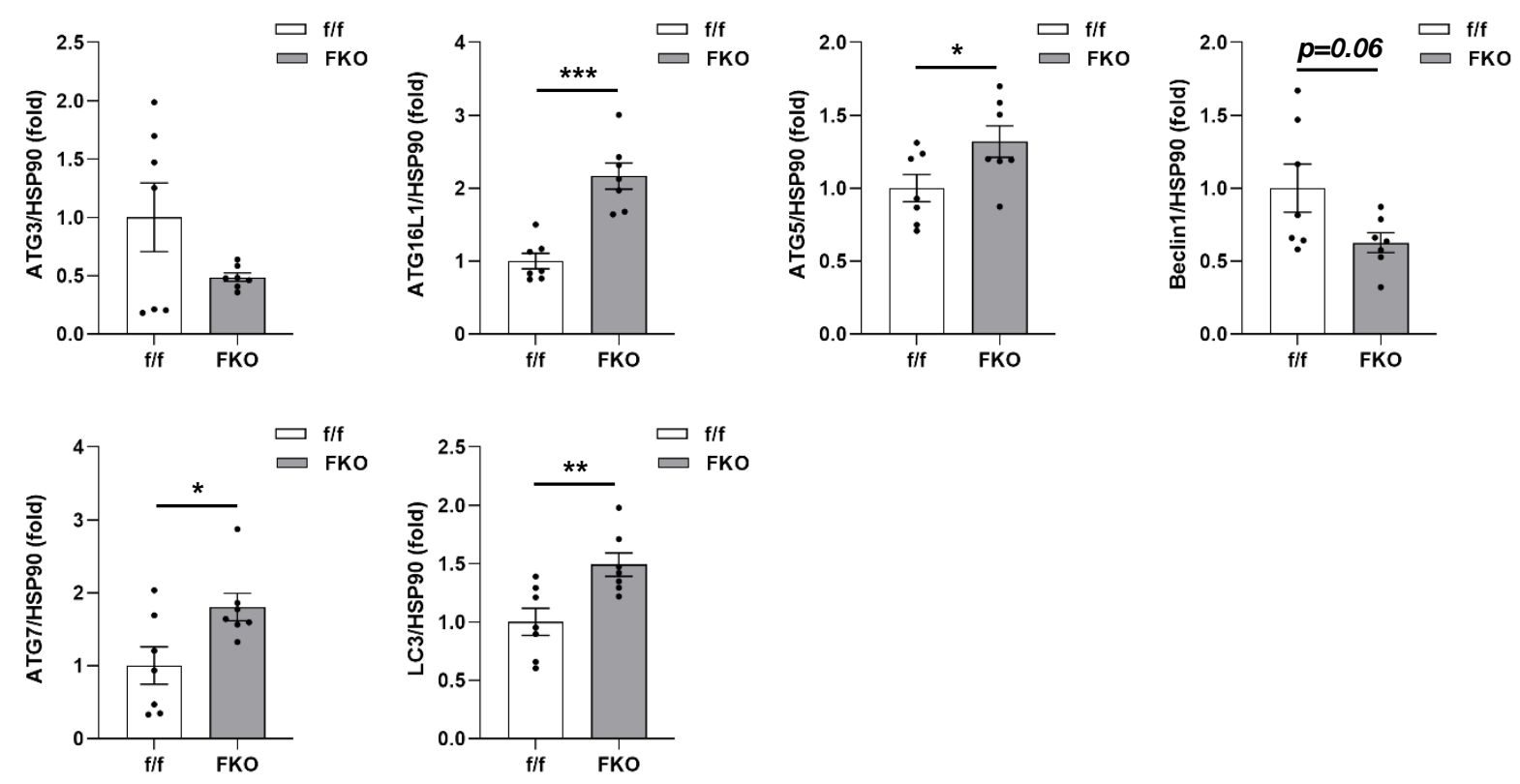


\section{Supplementary Fig. 8}

A
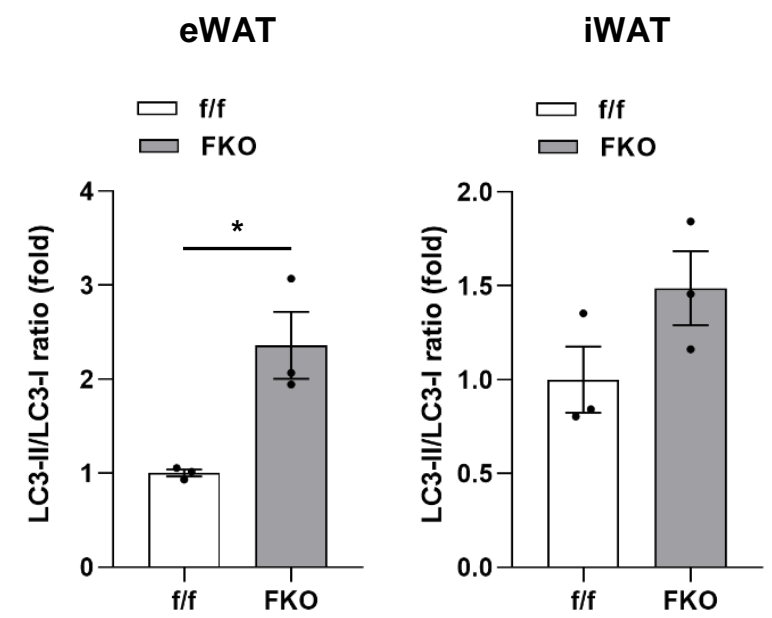

C

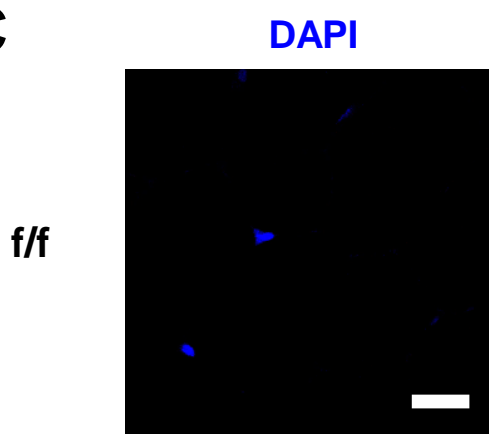

FKO
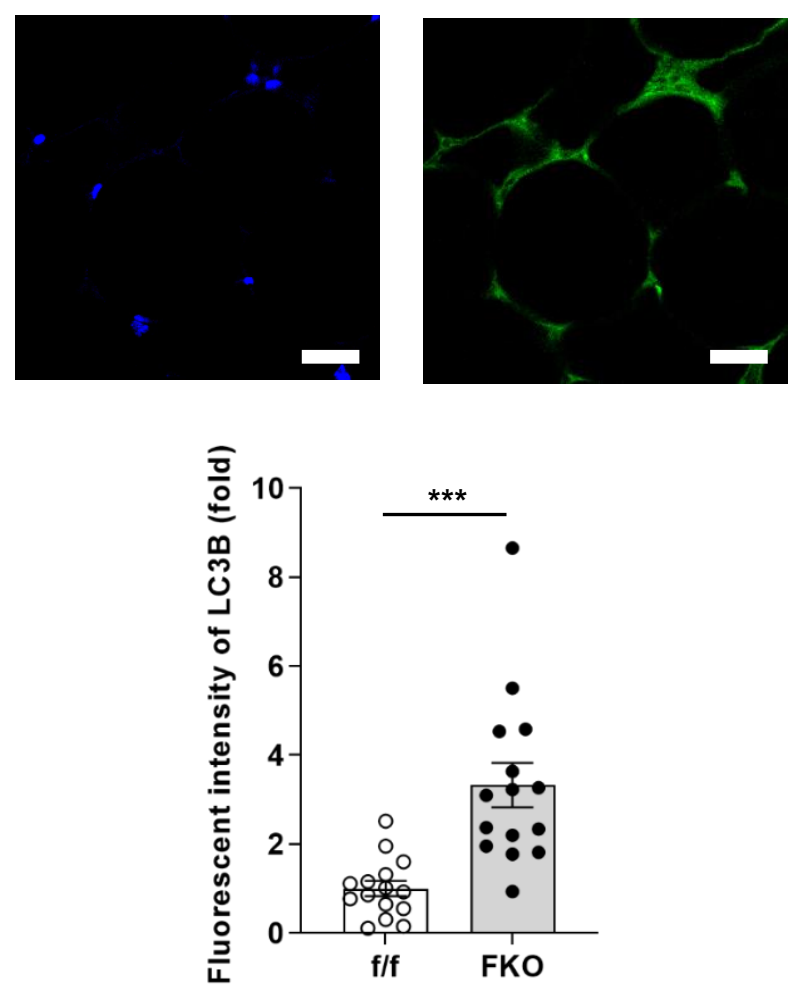

eWAT

B

LC3B

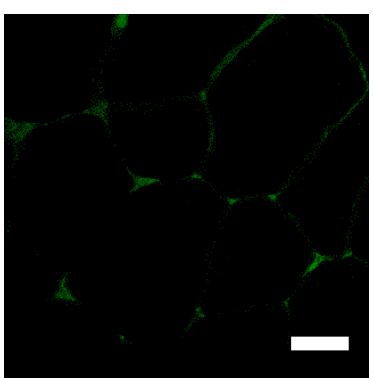

Liver

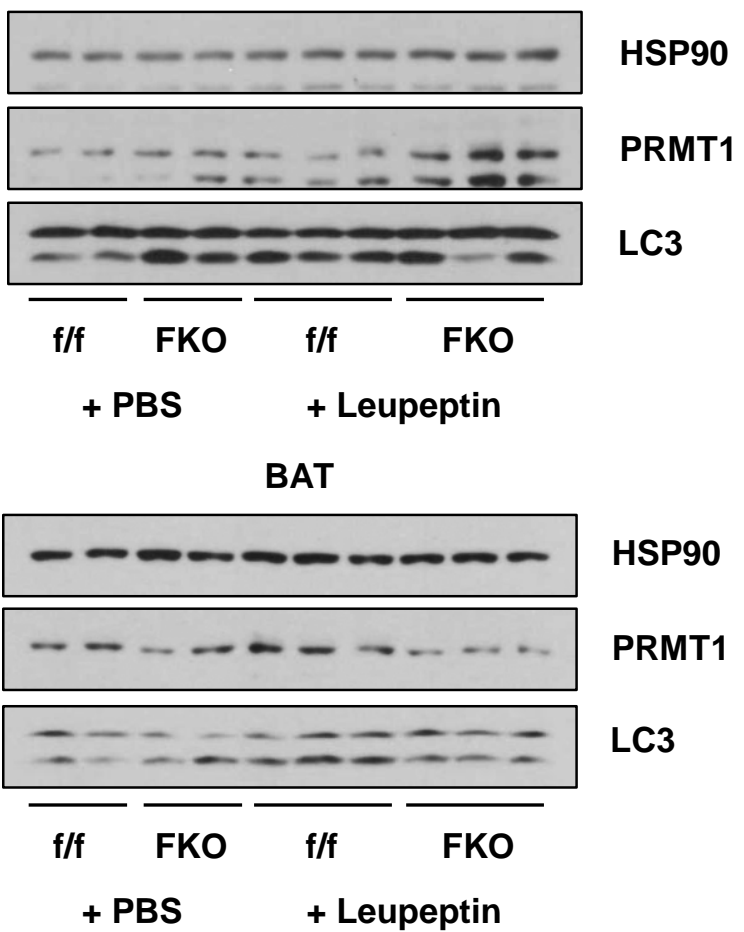

PLIN1

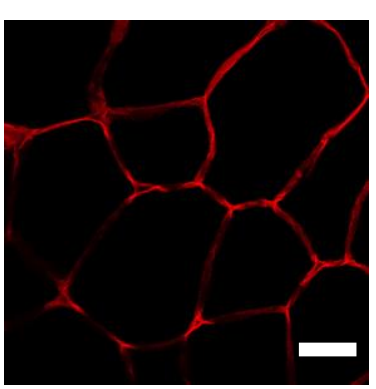

Merge
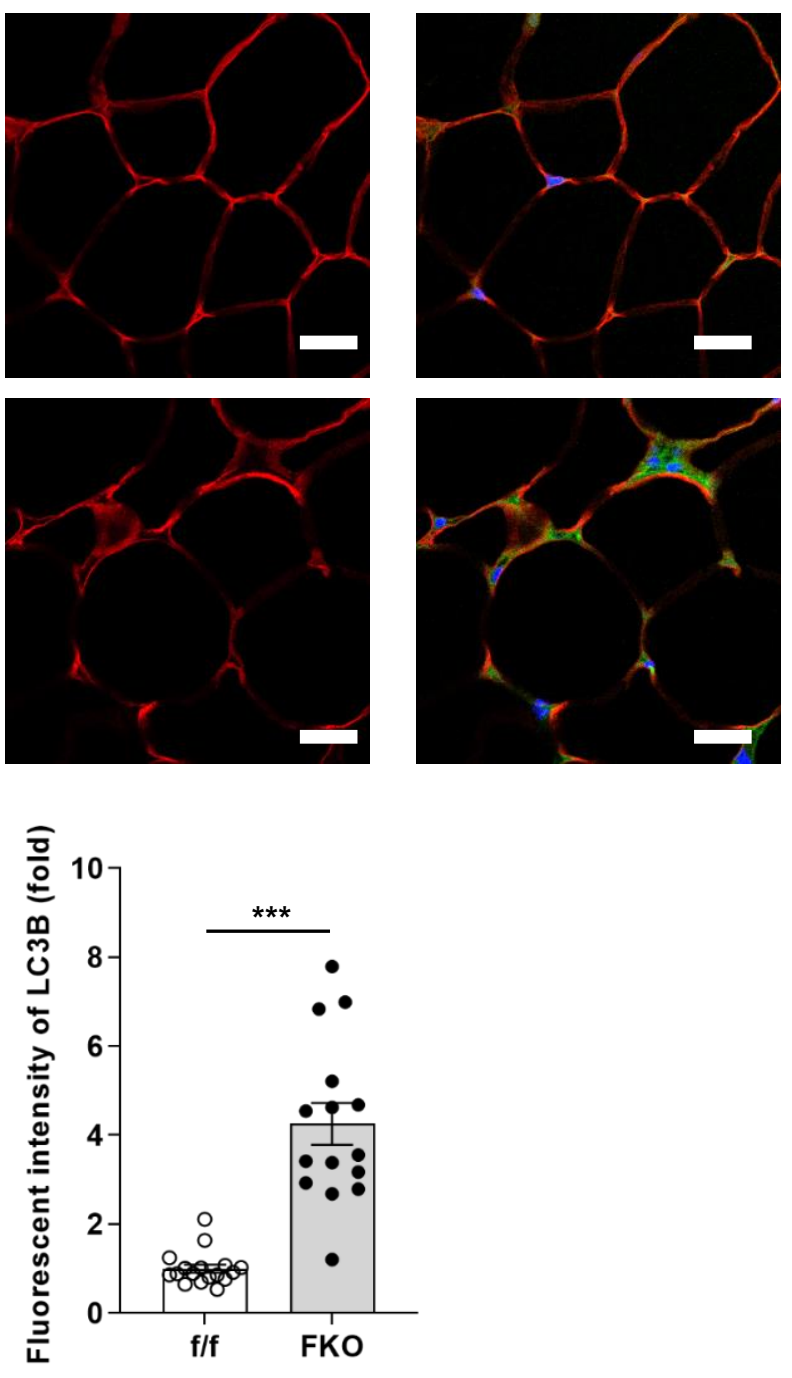

IWAT 
A

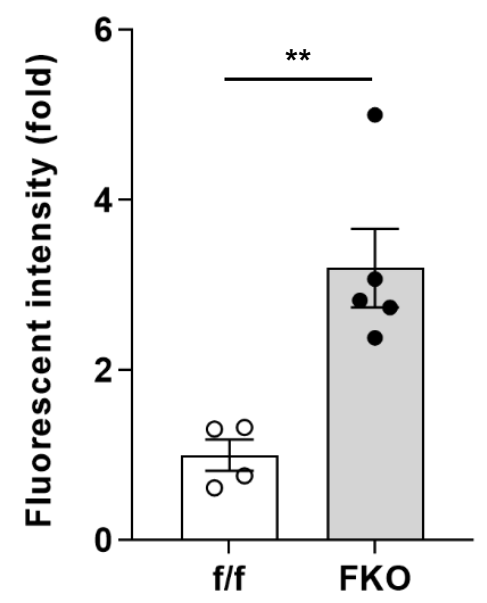

C

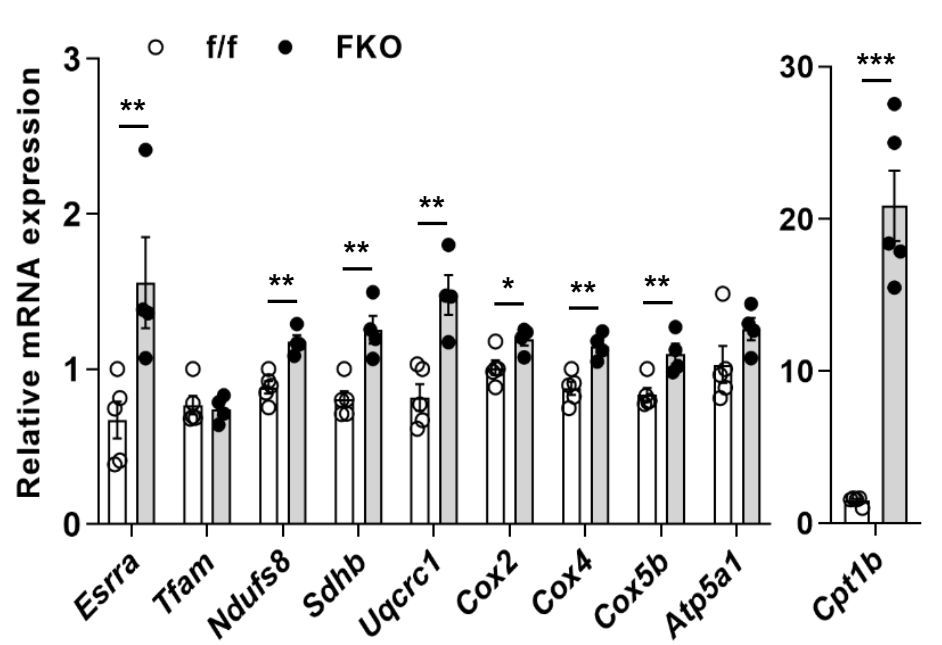

- f/f - FKo
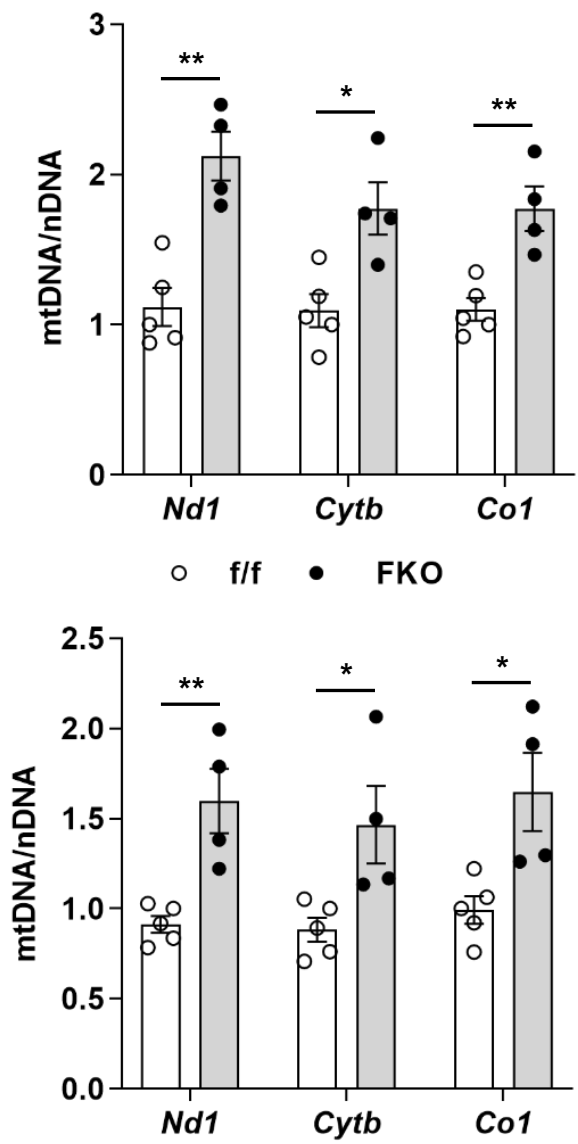

iWAT

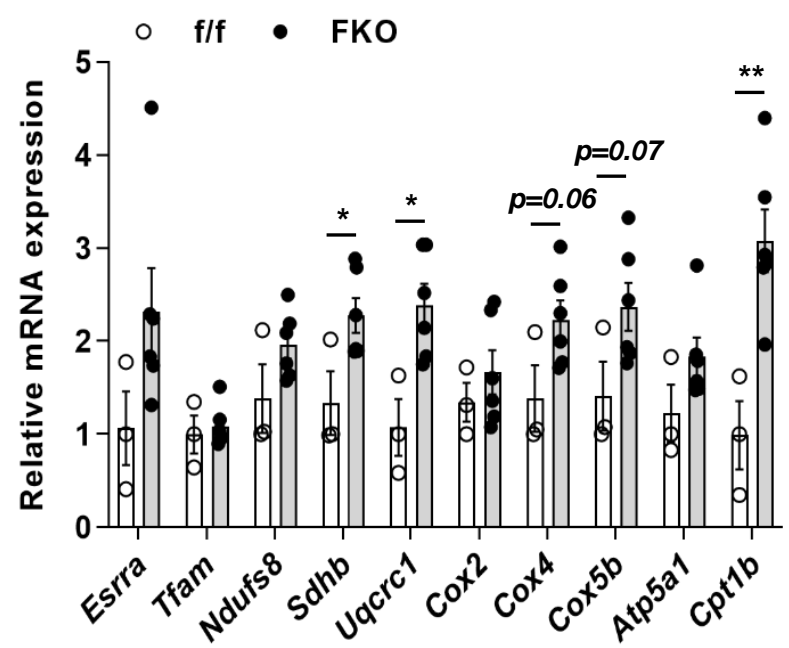




\section{Supplementary Fig. 10}
A
B
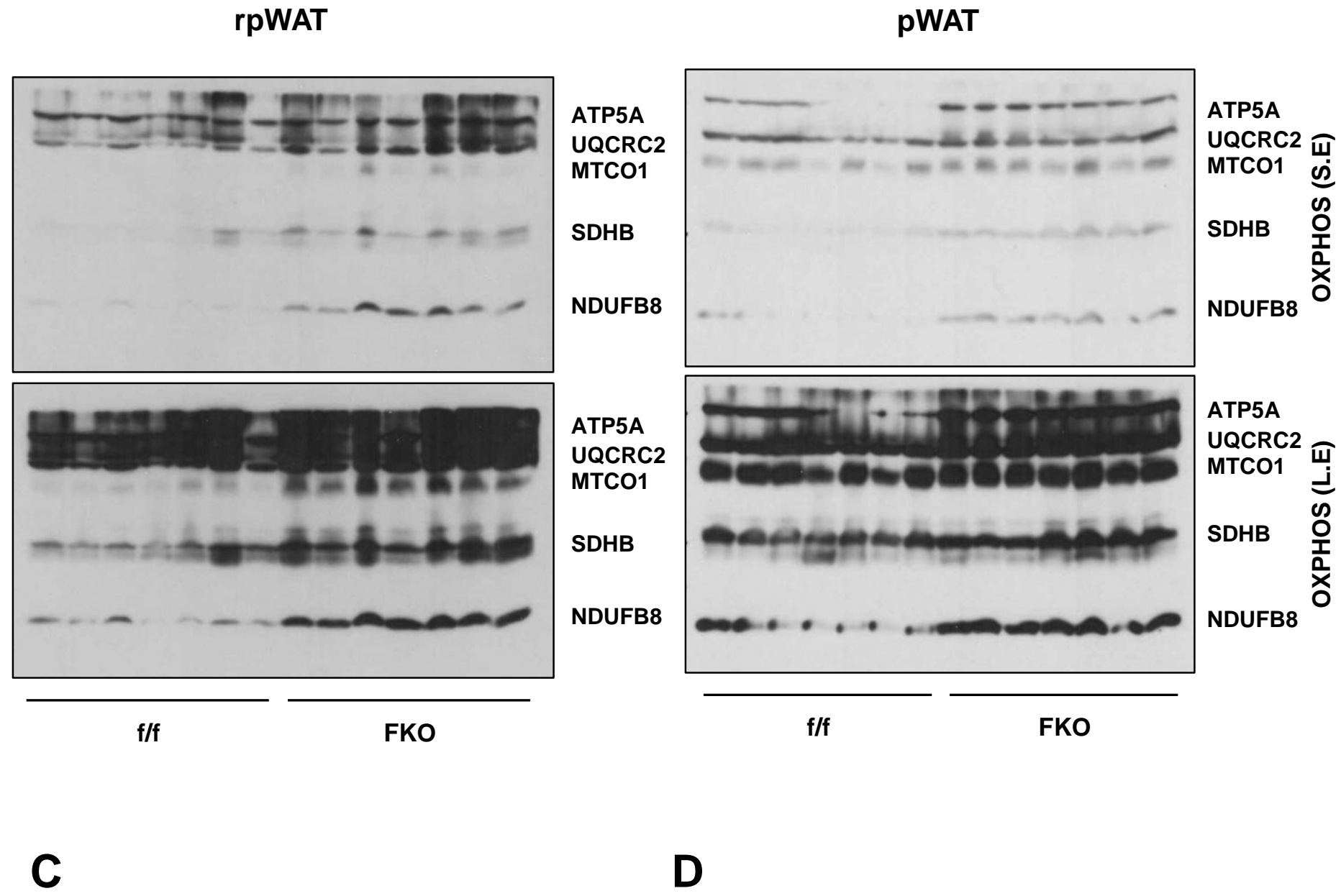

iWAT

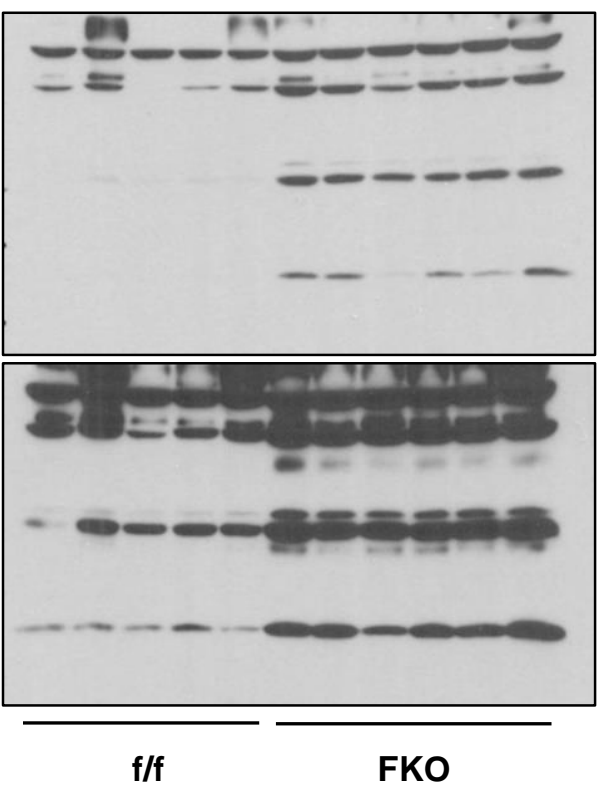

ATP5A
UQCRC2
MTCO1
SDHB
NDUFB8
ATP5A
UQCRC2
MTCO1
SDHB
NDUFB8

BAT

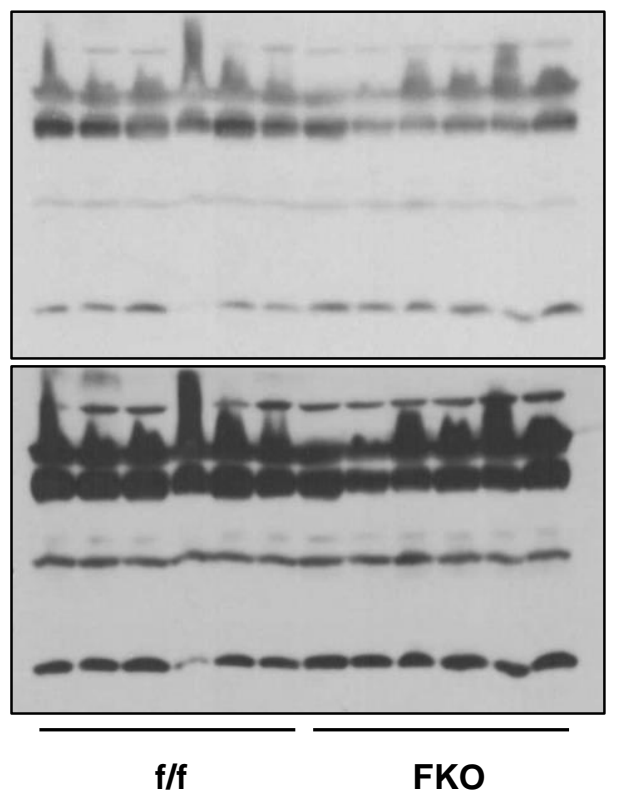

ATP5A

UQCRC2

MTCO1

SDHB

NDUFB8

(1)

NDUFB

ATP5A

UQCRC2

MTCO1

SDHB

NDUFB8

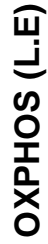




\section{Supplementary Fig. 11}

A
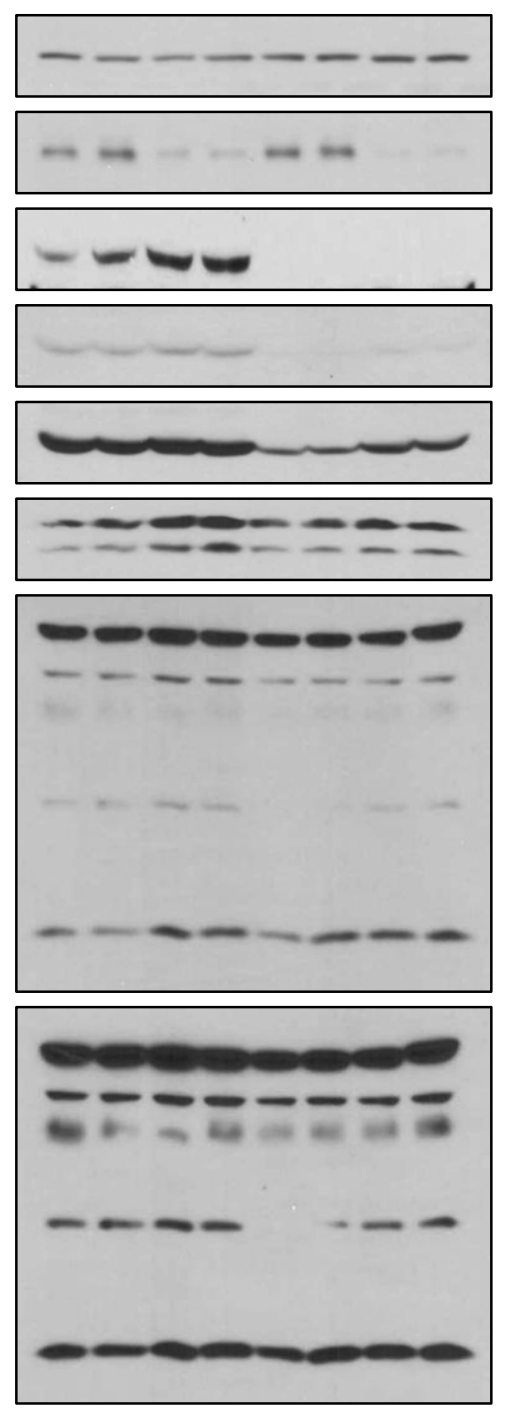

Con PR1 AMPKa PR1 + K/D K/D AMPKa K/D
B Differentiated primary adipocytes

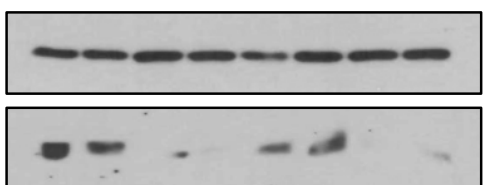

HSP90

HSP9O

PRMT1

AMPKa

p-AMPKa (S.E)

p-AMPKa (L.E)

\section{LC3}

\begin{tabular}{|c|c|}
\hline ATP5A & \\
\hline $\begin{array}{l}\text { UQCRC2 } \\
\text { MTCO1 }\end{array}$ & யூ \\
\hline SDHB & $\begin{array}{l}0 \\
0 \\
0 \\
0 \\
x\end{array}$ \\
\hline
\end{tabular}

ATP5A UQCRC2 MTCO1

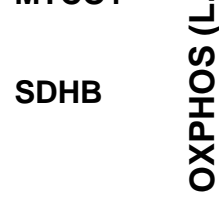

NDUFB8
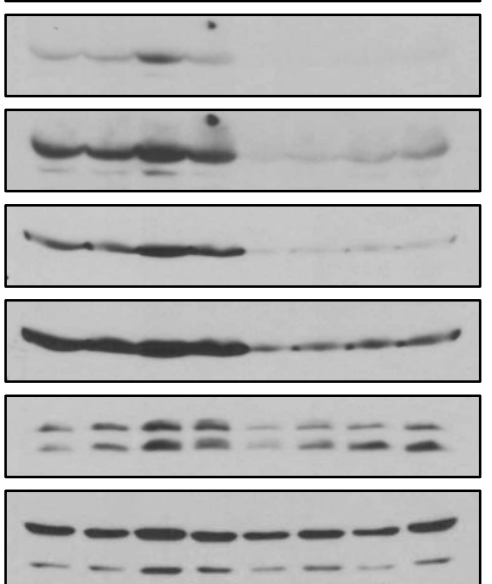

$$
\text { (1) }
$$
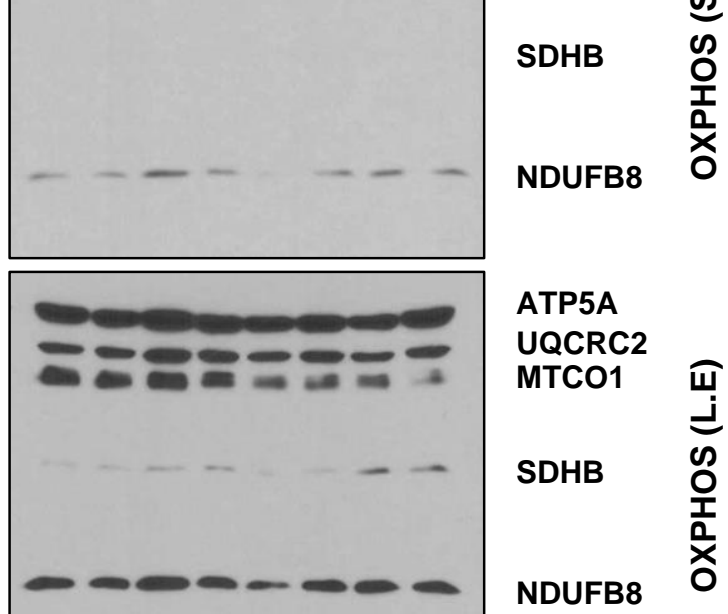

PRMT1

AMPKa (S.E)

AMPKa (L.E)

p-AMPKa (S.E)

p-AMPKa (L.E)

LC3

ATP5A

UQCRC2

MTCO1

SDHB

NDUFB8

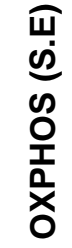

Con PR1 AMPKa PR1 +

K/D K/D AMPKa 


\section{Supplementary Fig. 12}

A
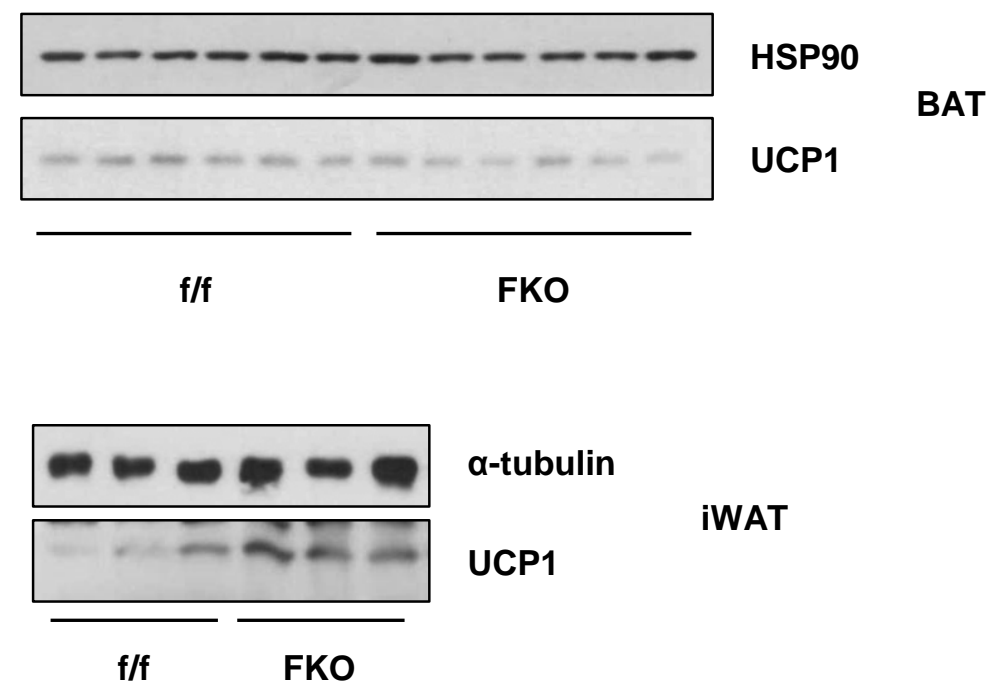

B

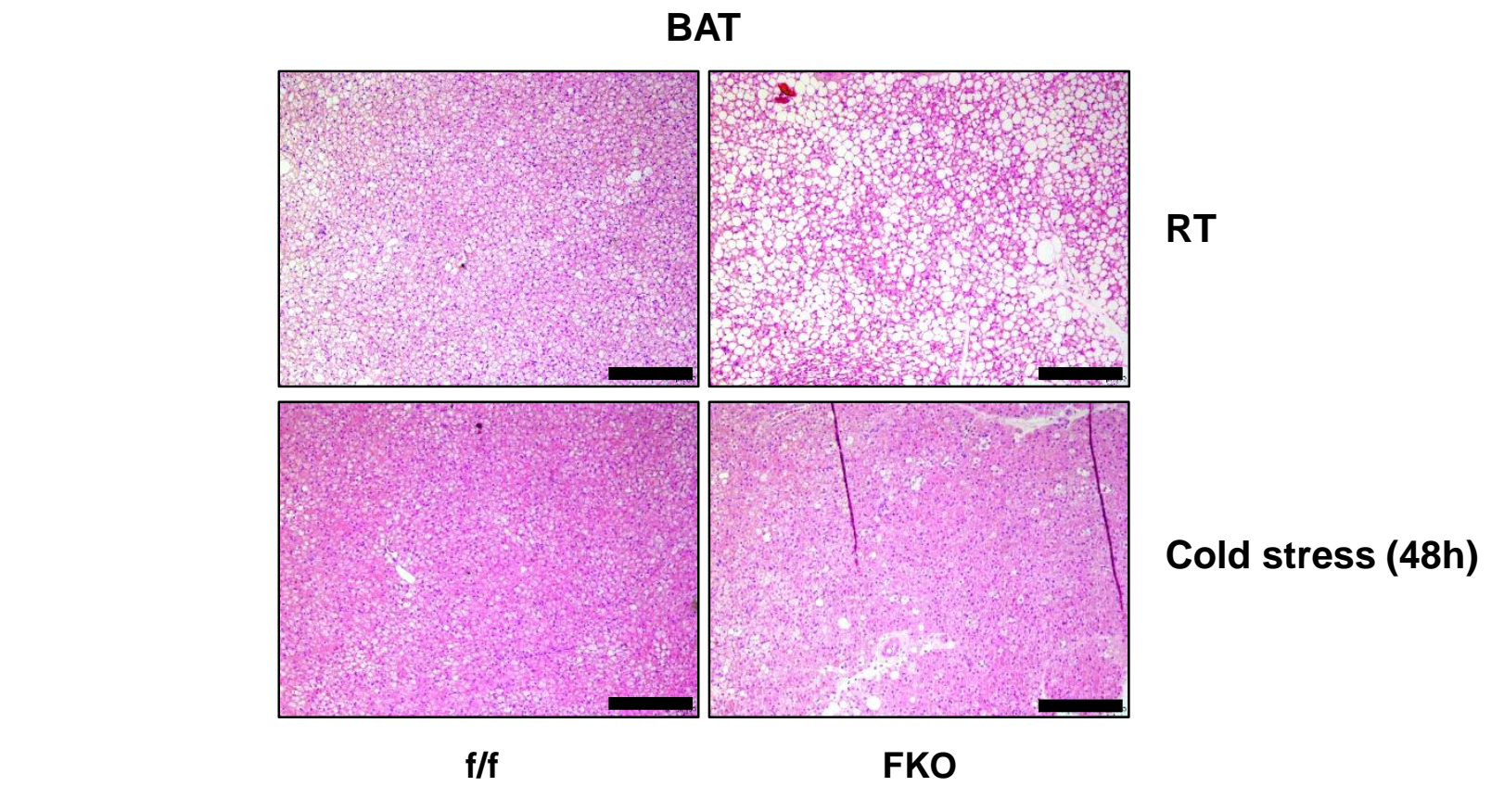

FKO 


\section{Supplementary Fig. 13}

A

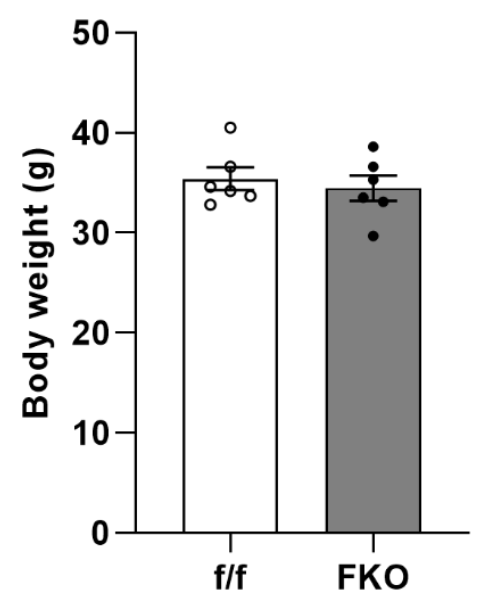

C

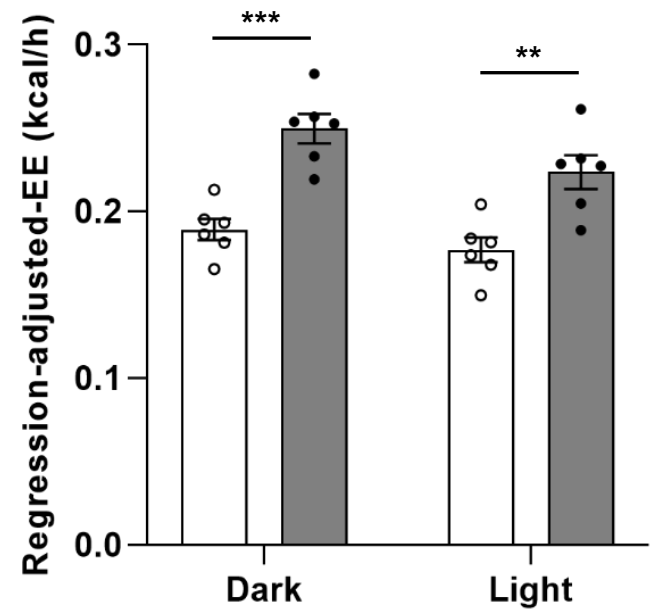

(Food intake)
B

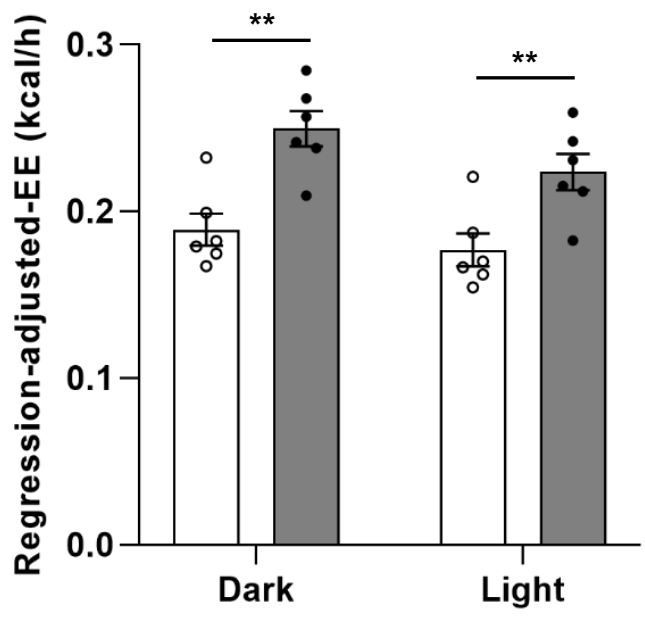

(B.W)

D

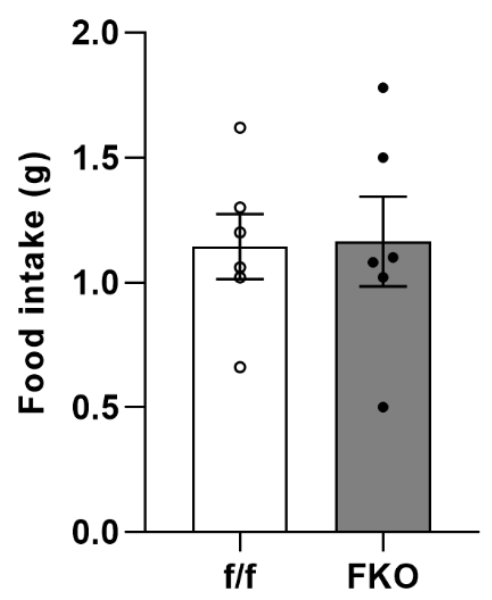




\section{Supplementary Fig. 14}

A
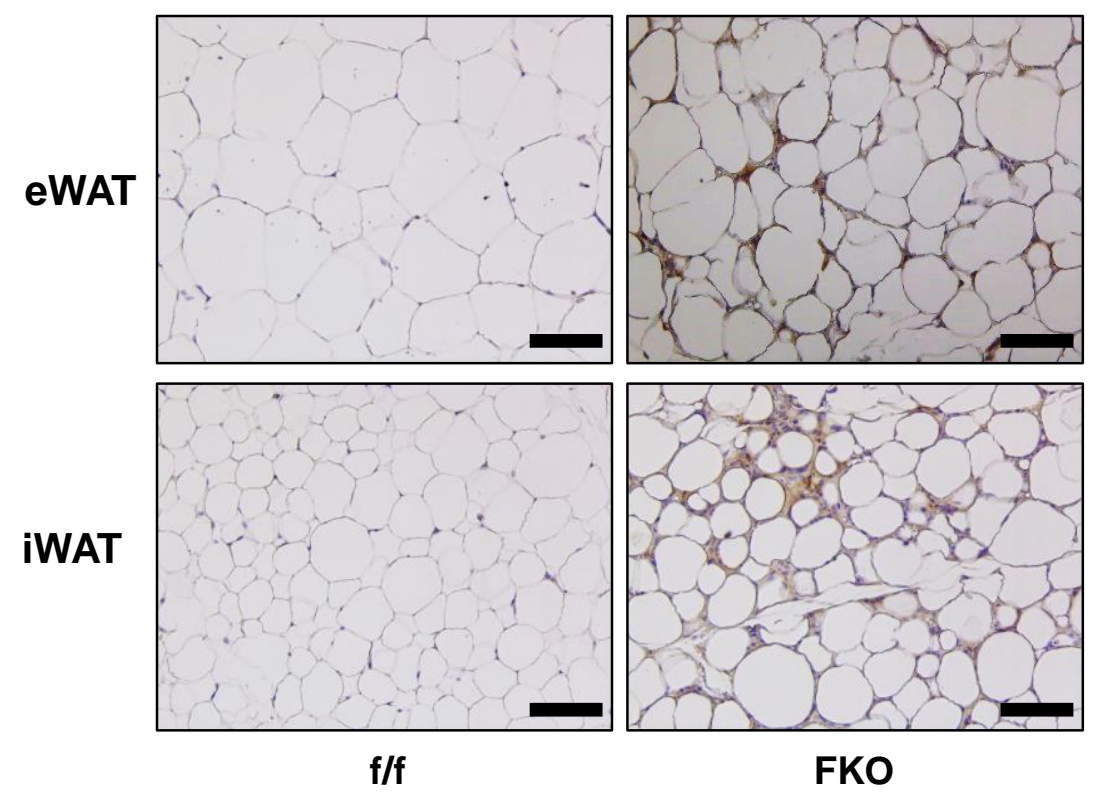

B

C

$\circ f / f \bullet F K O$
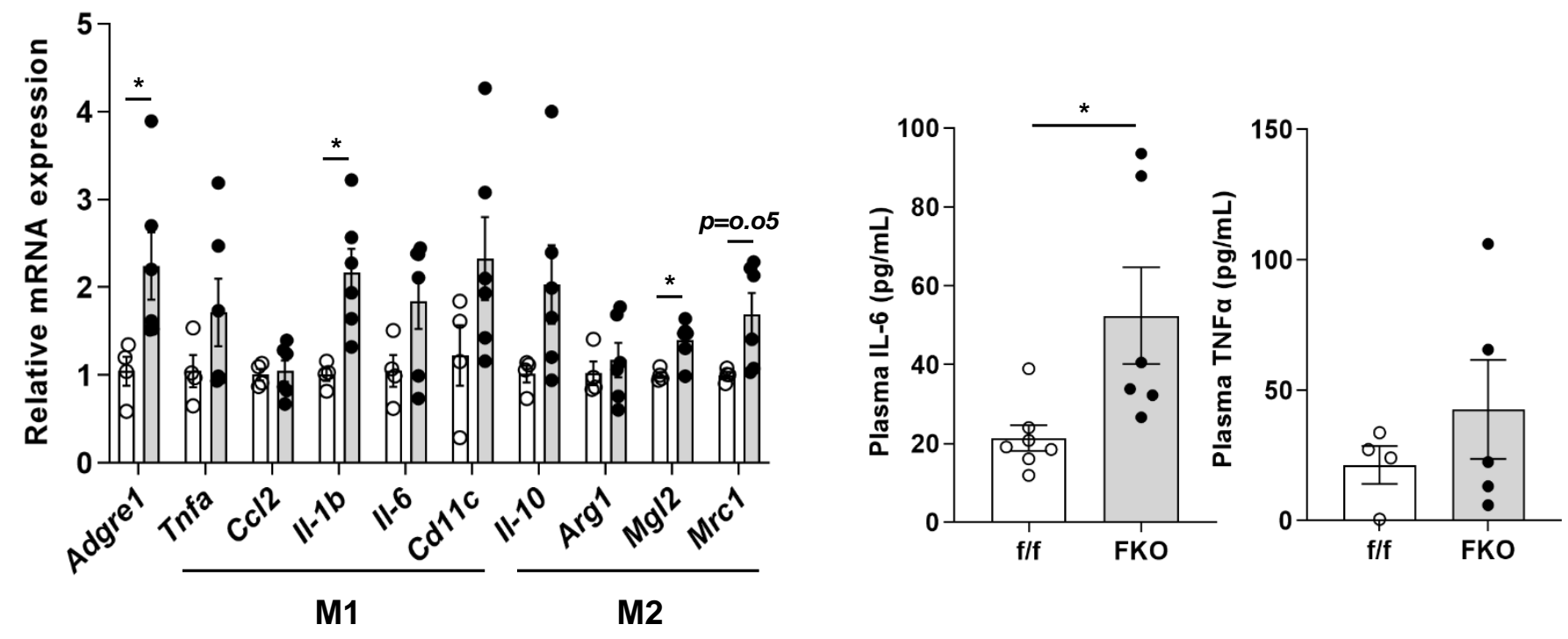


\section{Supplementary Fig. 15}

A ad lib. $\quad$ B

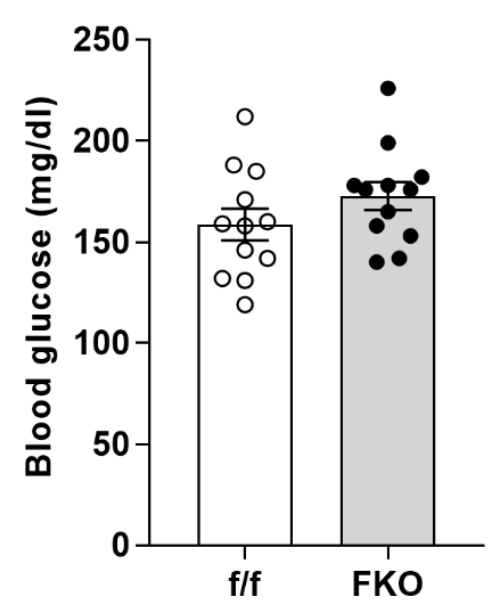

D
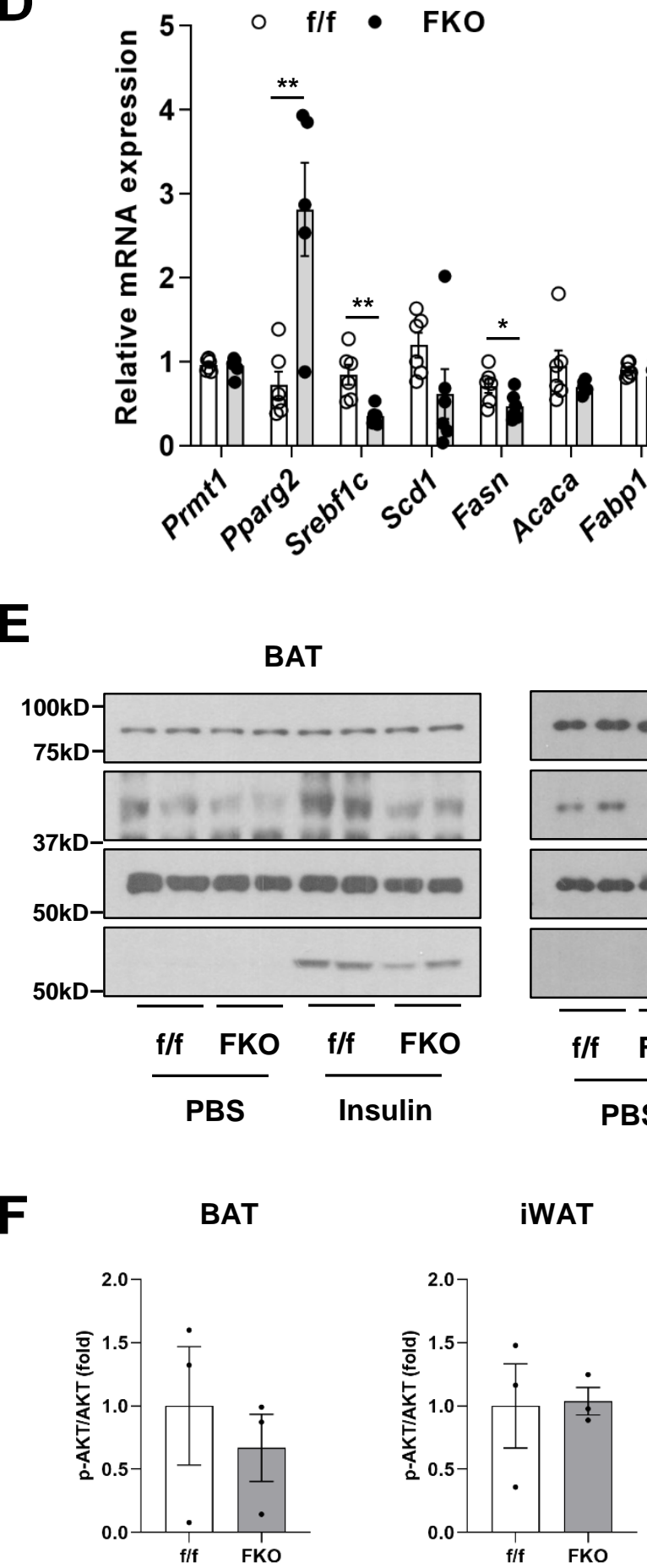

iWAT

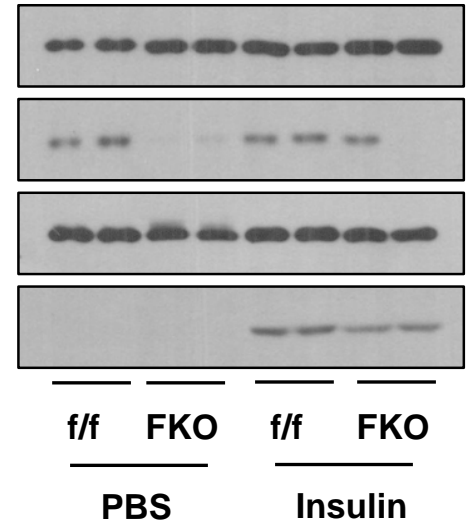

iWAT

Muscle

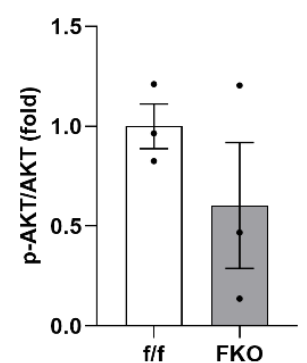

F

BAT

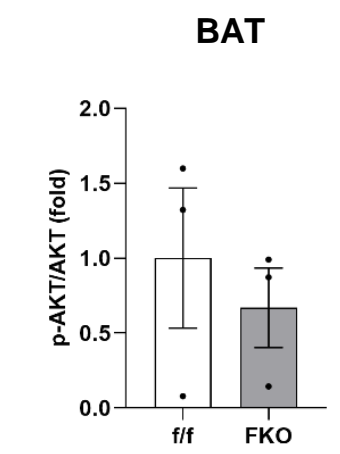

E

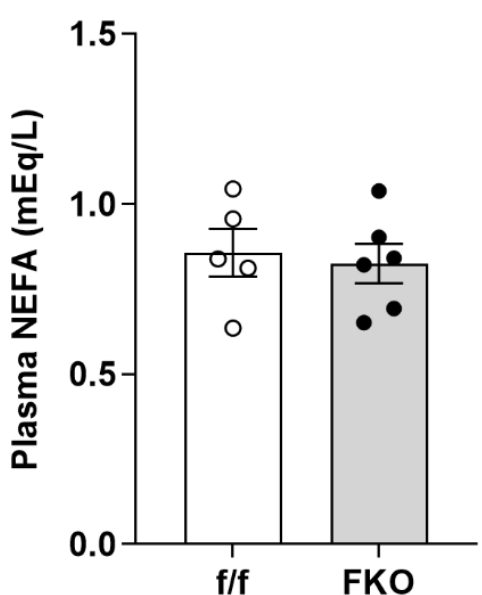

$\left\langle r^{2}\left\langle\theta^{2}\right.\right.$
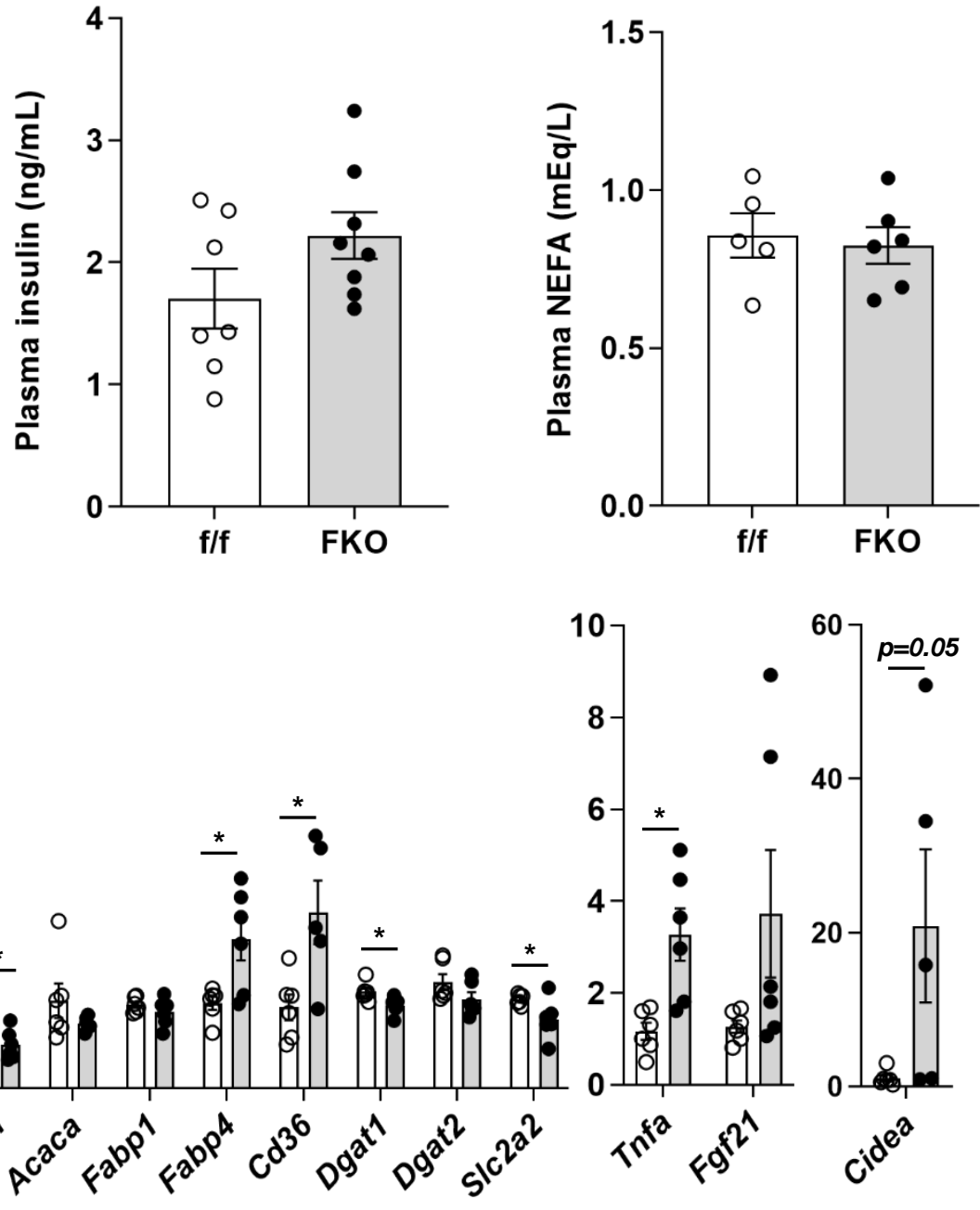
